\title{
Emulation of MIMO Rician-Fading Environments With Mode-Stirred Reverberation Chambers
}

\author{
Juan D. Sánchez-Heredia, Juan F. Valenzuela-Valdés, Antonio M. Martínez-González, and \\ David A. Sánchez-Hernández, Senior Member, IEEE
}

\begin{abstract}
Some recent publications have extended the emulating capabilities of mode-stirred reverberation chambers, which are now able to emulate Ricean-fading and non-isotropic environments. Either the need to physically modify existing chambers or multiple sets of measurements is required for these enhancements. In this paper a novel post-processing hybrid tool is presented for the transformation of a Rayleigh-fading emulated environment into a Rician one. The model is tested and compared to outdoor measurements and simulations through the K-factor, correlation, diversity gain and MIMO capacity. Results show an excellent matching performance with no hardware modifications of mode-stirred chambers with just one set of measurements. The method is patent protected by EMITE Ing.
\end{abstract}

Index Terms-Channel capacity, diversity gain, multiple-inputmultiple-output (MIMO) systems, radiation efficiency.

\section{INTRODUCTION}

A single-cavity mode-stirred reverberation chamber, also known as a reverberation chamber (RC), is a metal cavity sufficiently large to support many resonant modes. Although RCs have been extensively used in electromagnetic compatibility problems [1], their use for antennas and propagation-related problems have only acquired attention in the last few years. This is due to their ability to artificially generate a repeatable multipath environment. RCs were initially thought to provide an isotropic and randomly polarized measuring scenario. This was done by perturbing the modes with stirrers and rotating platforms. In this way a fading environment similar to the ones found in indoor and urban environments (Rayleigh) but with a uniform elevation distribution of the incoming waves is obtained [2], [3]. The real and imaginary parts of the received signal become then normally (Gaussian) distributed, with the associated magnitude following a Rayleigh distribution and the phase following a uniform distribution over

Manuscript received October 26, 2009; revised June 16, 2010; accepted October 25, 2010. Date of publication December 03, 2010; date of current version February 02, 2011. This work was supported in part by Fundación Séneca, the R\&D unit of the Autonomous Region of Murcia (Spain) under project reference 11783/PI/09 and in part by the Spanish National R\&D Programme through TEC2008-05811.

J. Valenzuela-Valdés is with the EMITE Ingeniería SLNE, Edificio CEEIM, E-30100 Espinardo, Murcia, Spain (e-mail: juan.valenzuela@emite-ingenieria. es).

J. D. Sánchez-Heredia, A. M. Martínez-González, and D. A. SánchezHernández are with the Departamento de Tecnologías de la Información y Comunicaciones, Universidad Politécnica de Cartagena, Cartagena E-30202, Spain (e-mail: david.sanchez@upct.es).

Color versions of one or more of the figures in this paper are available online at http://ieeexplore.ieee.org.

Digital Object Identifier 10.1109/TAP.2010.2096185
$2 \pi$. While avoiding cumbersome outdoor measurements, compact-size RCs provide accurate, repeatable and reliable ways of validating measurements for antenna systems and diversity schemes [4]. Yet, real propagating scenarios rarely follow an ideal Rayleigh-fading environment. The K-factor of more general Rician-fading environments changes as the distance of receiver to transmitter changes [5]. Macrocells usually offer a greater K-factor than microcells [6] while rich multipath environments provide $\mathrm{K}$-factors typically close to 0 [7]. Consequently, recent research efforts have concentrated on extending the original capabilities of first-generation RCs. Good examples are the recent extension to non-isotropic environments [8] and the emulation of the effects of metallic windows and other artefacts, trees and walls in buildings [9], although this last one only for Rayleigh-fading scenarios. Unlike reverberation chambers (RCs), where only one cavity is used, MSCs may contain more than one metal cavity. Cavities are coupled by diverse means and a rich fading environment consisting on diverse clusters with different fading characteristics can be obtained. The MSC also contains a set of mode stirrers that change the boundary conditions of the main cavity within the chamber. This causes a multi-reflective environment which is repeatable and can be statistically studied. MSCs can be used as a very fast, easy, and accurate tool to measure a wide variety of MIMO parameters for different extension of the performance of conventional RCs [10]-[12]. With the use of appropriate equipment and through adequate processing of the measured scattering (S) parameters, radiation efficiency, self-impedance, total radiated power, total isotropic sensitivity and several MIMO channel performance variables, including bit error rate (BER), can now be evaluated using MSCs. The extension to emulate Rician-fading [13] has also been available recently. In this Rician-fading emulation technique, however, either complex hardware alterations or large sets of measurements are required. In [13], the Rician-fading environment is obtained at the cost of varying the chamber characteristics and/or the antenna configuration. There is an inherently higher hardware complexity to this option.

In this paper, a novel mode-processing technique which allows for an emulation of Rician-fading environments from the data taken in a mode-stirred reverberation chamber with Rayleigh-fading emulated environment is presented. The method is not intended to describe in detail the response of a device to a change in the angle of arrival (AoA), but rather uses a deterministic component of a Rician signal via post processing to observe the associated changes in the conventional post-processing techniques used to determine general MIMO parameters of the device, such as diversity gain and MIMO 


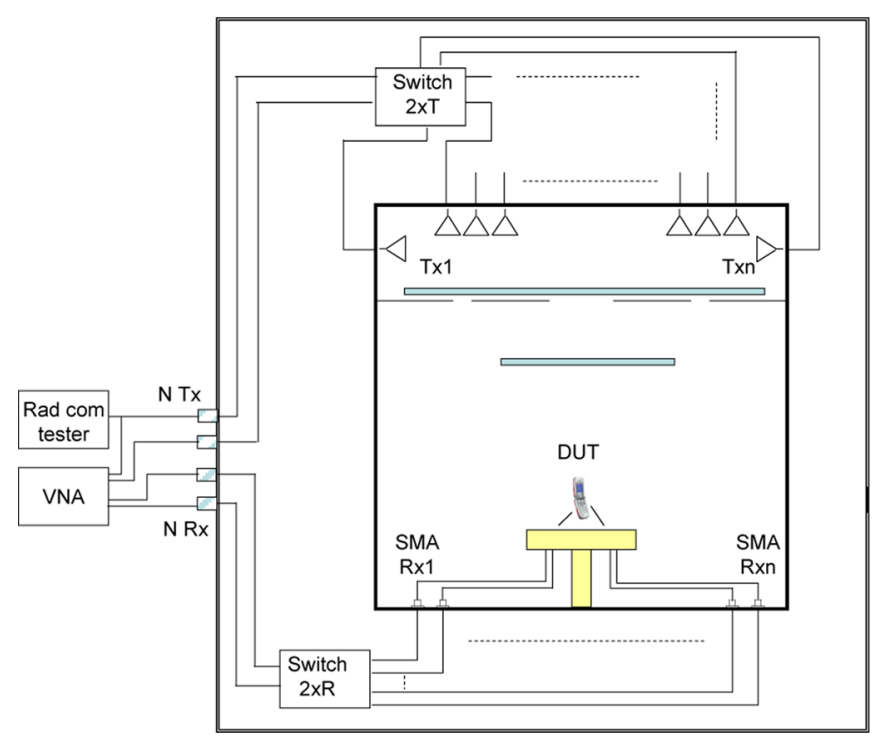

Fig. 1. Sketch of measurements with the E200 MIMO analyzer.

capacity. The method allows the MIMO designer to evaluate several Rician K-factors environments with a single measurement set in the MSC. With this technique a considerable reduction of chamber cost and complexity as well as measuring time is provided, alleviating the hardware complexity of other techniques. Section II describes the proposed measurement and emulation technique for Rician-fading environments. The method is validated through a comparison to other simulated and measured results. Channel eigenvalues, correlation, diversity gain and system capacity results for several scenarios are described in Section III. Finally, some conclusions are outlined in Section IV.

\section{Emulating AND MEAsuring Technique}

Measurements illustrated in this paper have been performed with the EMITE Ing $8 \times 8$ MIMO Analyzer Series E200a in connection to the Rohde \& Schwarz ZVRE Vector Network Analyzer $(9 \mathrm{kHz}$ to $4 \mathrm{GHz})$. The MIMO Analyzer is a second generation two-cavity mode-stirred reverberation chamber with external dimensions of $0.82 \mathrm{~m} \times 1.275 \mathrm{~m} \times 1.95 \mathrm{~m}, 8$ exciting antennas, polarization stirring due to aperture-coupling and to the different orientation of the antenna exciting elements, 3 mechanical and mode-coupling stirrers, 1 holder-stirrer and variable iris-coupling. The MIMO Analyzer was set-up for 3 holder positions with 15 different mechanical stirrer positions for each holder position, 12 iris-coupling aperture stirring and $20 \mathrm{MHz}$ frequency stirring. Measurements were performed at $1800 \mathrm{MHz}$ and half-wave dipoles were used as MIMO antennas. A sketch of the measurement setup can be observed from Fig. 1.

The K-factor, defined as the ratio of the direct path component to the scattered component can be calculated from measured S-parameter in a mode-stirred reverberation chamber by [13],

$$
K=\frac{\text { direct component }}{\text { scatterd components }}=\frac{d_{R}^{2}}{2 \sigma_{R}^{2}}=\frac{\left(\left|\left\langle s_{21}\right\rangle\right|\right)^{2}}{\left\langle\left|s_{21}-\left\langle s_{21}\right\rangle\right|^{2}\right\rangle}
$$

where $S_{21}$ is the measured parameter in the mode-stirred reverberation chamber for each antenna pair, $\sigma_{R}$ is the radius of cluster data and $d_{R}$ is the distance of centroid of cluster from the origin. A scatter plot would result in the data clustered in a circle and centered about the origin for pure Rayleigh-fading environments $(K=0)$. As the direct line of sight (LoS) energy became comparable to the non-LoS energy, the cluster of data would move away from the origin, and the fading environment becomes a Rician one. Scatter plots are useful not only for identifying the LoS behavior of the fading environments, but also to quantify the data dispersion through the K-factor. The data set measured with the chamber has to be large enough to ensure a Rayleigh-fading scenario. While this is a typical use of RCs, it also means that both the direct and non-direct coupling paths are included in the data set in a more complex mode-coupling muticavity MSC. It is clear from (1) that by increasing the direct component the $\mathrm{K}$-factor will increase. Consequently, we can move the cluster data away from the origin by adding an offset continuous component. The offset has to be a complex number by

$$
\begin{array}{r}
\text { off set }=\mid \text { offset }\left.\right|^{*} \cos \left(\text { off } \text { set }_{\varangle}\right) \\
+i^{*} \mid \text { off set }\left.\right|^{*} \operatorname{sen}\left(\text { off set } t_{\varangle}\right) .
\end{array}
$$

For a target K-factor $\left(K_{\text {target }}\right)$, the required offset can be defined by

$$
\mid \text { off set } \mid=\sqrt{K_{\text {target }}{ }^{*} 2 \sigma_{R}^{2}-\left|d_{R}\right|} .
$$

The phase of the offset in (3) is obtained from the averaged phase of all data samples in the initial set. Equation (3) can be re-written in terms of the S-parameters by

$$
\mid \text { off set }\left|=\sqrt{K_{\text {target }}{ }^{*} 2\left\langle\left|s_{21}-\left\langle s_{21}\right\rangle\right|^{2}\right\rangle}-\right|\left\langle s_{21}\right\rangle \mid .
$$

In order to obtain the desired results, the added offset would have to be phase-coherent to the selected radius in the way

$$
\text { off set }{ }_{\varangle}=d_{R \varangle}=\left\langle s_{21}\right\rangle_{\varangle} .
$$

The calculated offset has to be added to all samples that are used for the Rician emulation. In this way the new Rician-fading samples keep the standard deviation of the original Rayleighfading ones, and therefore the distance can be altered to be adapted to the target K-factor. Fig. 2 depicts several scatter plots for the original Rayleigh-fading samples and different Ricianfading ones with $K=5,15$ and 100 using the proposed technique.

It is clearly observed from this figure that the modified samples keep the standard deviation while their radius varies in a proportional way to the target K-factor. Fig. 3 shows a comparison between measured histograms for diverse sample sets with their associated probability distribution functions and several emulated sample sets. Originally-emulated samples can clearly be associated to a Rayleigh-fading scenario, while modified samples conform very well to measured Rician-fading 


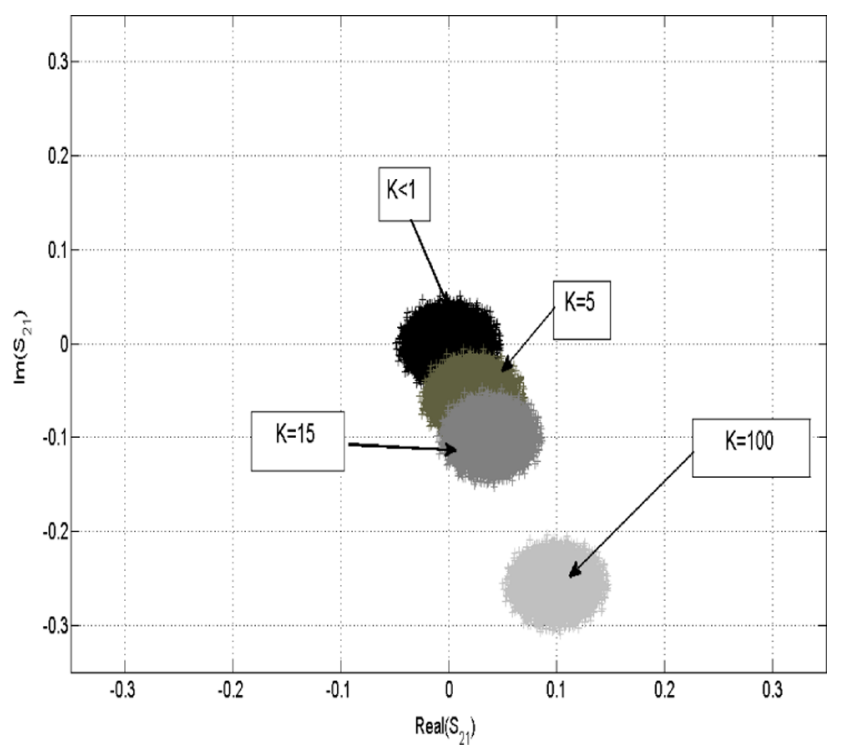

Fig. 2. Scatter plots for mode-stirred and mode-processed data.

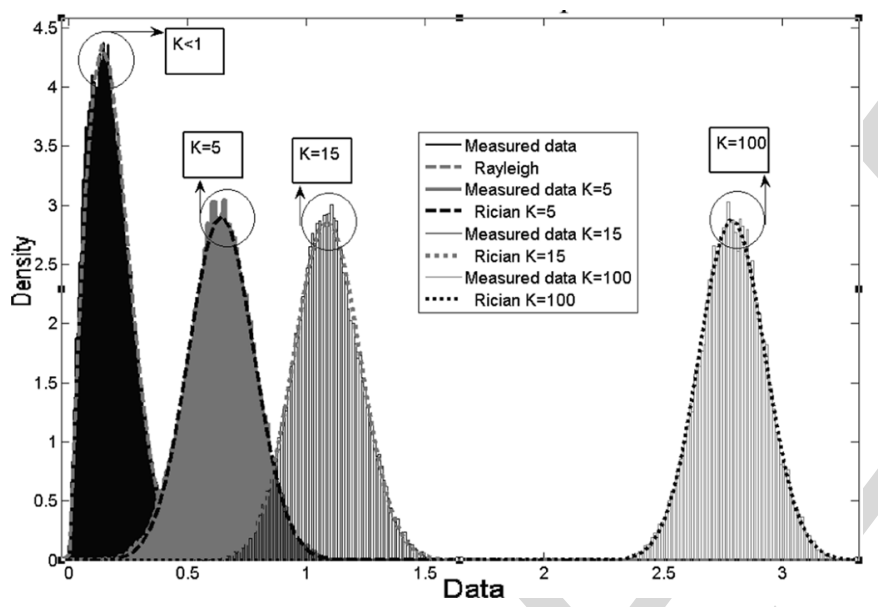

Fig. 3. Histograms for measured and modified data.

scenarios with $K=5,15$ and 100. It seems clear, however, that the method relies on the previous estimation of the initial $K$ factor of the sample set. Consequently, the uncertainties of this method are linked to the uncertainties of the method employed to estimate the initial $K$ factor of the sample set.

In order to validate the proposed technique, 3 different initial $3 \times 3$ MIMO systems were measured. Three $\lambda / 2$ dipoles in a vertical position were employed as antennas in reception. The difference between the tested arrays as the spatial separation between the receiving dipoles, as shown in Table I. The initial measured K-factor was always below 0.0001 for the 3 systems, that is, clearly a Rayleigh-fading environment. The initial K-factors were then incremented slightly to between $10^{-3}$ and $10^{3}$ using the proposed technique. In this way, with only 3 system measurements in a Rayleigh-fading environment, it was possible to emulate and evaluate their performance for any Rician-fading environment. This drastically reduces both R\&D costs and time. The emulated results were compared with the outdoor measurements results in [14]. This comparison is shown

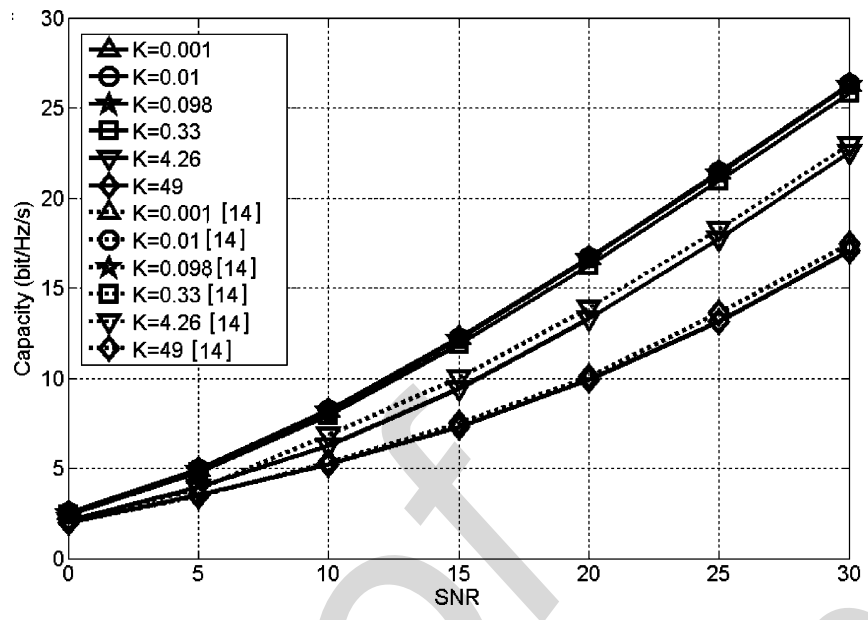

Fig. 4. Measured and emulated capacity versus SNR for $3 \times 3$ MIMO systems

TABLE I

MEASURED MIMO ARRAYS

\begin{tabular}{|c|c|c|}
\hline System & $\begin{array}{c}\text { Spatial separation between } \\
\text { adjacent antennas }(\mathrm{d} / \lambda)\end{array}$ & $\begin{array}{c}\text { Initial measured K- } \\
\text { factor }\end{array}$ \\
\hline $\mathrm{A}$ & 0.01 & $<0.0001$ \\
\hline $\mathrm{B}$ & 0.05 & $<0.0001$ \\
\hline $\mathrm{C}$ & 0.10 & $<0.0001$ \\
\hline
\end{tabular}

in Fig. 4. Good agreement is observed between outdoor measurements and measurements emulated with the proposed technique. Thus, this method does not intend to describe the specific response of the device to a change in the specific angle of arrival (AoA) characteristic of a fading scenario, but rather to describe the changes in the general performance of the device when the scenario contains an increased LoS component. In Fig. 4, the method closely follows the real evaluation of a device for changing $\mathrm{K}$ factors in outdoor measurements made in [14]. This is clear evidence that the technique represents a step forward to the goal of obtaining real-world performance from a mode-stirred chamber test.

\section{MEASURED RESUlts}

\section{A. Correlation Results}

A traditional parameter to be analyzed is the correlation between antennas, which gives us an idea of signal similarities. This parameter has been postulated as sufficient to characterize the MIMO systems in some popular models such as the Kronecker model [15]-[18]. Recent analyses, however, have raised the need of more information to accurately predict system capacity [19], [20]. With the aid of the proposed technique, the correlation properties in terms of the fading environment could be studied. In this way we can analyze how does the correlation coefficient change from Rayleigh-fading environments $(K=0)$ as $\mathrm{K}$-factors are increased. Fig. 5 shows the correlation coefficient between adjacent antennas for the different systems in Table I. From this figure one can observe that different correlation characteristics exist for the different systems. As it was expected, the system with highest correlation coefficient is system 


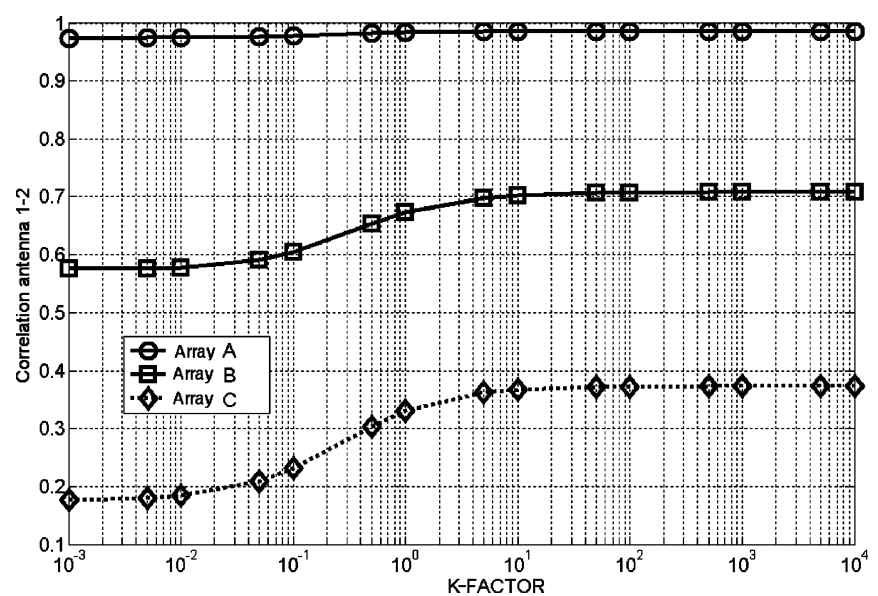

Fig. 5. Emulated correlation coefficients between adjacent antennas for the MIMO systems in Table I.

A. This is because of its smallest separation distance between adjacent vertically-polarized dipole antennas, which leads to the highest correlation coefficient. In consequence, system $\mathrm{C}$ depicts the lowest correlation coefficients. It is also important to note from Fig. 5 that the correlation coefficients change with changes in the $\mathrm{K}$-factor. While the increment of correlation in system A with increasing $\mathrm{K}$ factor is almost imperceptible $(0.97$ to 0.98 ), system $C$ nearly doubles this parameter (0.18 to 0.39 ), while system B provides for a $22.4 \%$ increment $(0.58$ to 0.71$)$. Therefore, one can conclude that when increasing the K-factor the correlation coefficient also increases, and this increment is more noticeable when the correlation factor is low. In other words, the influence of the K-factor is more important for systems with low correlation between adjacent antennas. It is also important to stress that the increase in the change in the correlation coefficient that occurs when varying the $\mathrm{K}$-factor has a limitation. For K-factors below 0.01 the correlation remains constant. For K-factors between 0.01 and 0.1 the correlation increases very quickly with increasing K-factor. This K-factor range accounts for $10 \%$ of the total correlation increment. For $\mathrm{K}$-factors between 0.1 and 10 , the correlation increment slope is more pronounced, achieving in this $\mathrm{K}$-factor range a $90 \%$ of the total increase in correlation. Finally, for K-factors above 10 the correlation coefficient again remains constant. This means that the environment influences the correlation factor in a specific $\mathrm{K}$-factor range. In other words, the $\mathrm{K}$-factor plays a role on correlation coefficients for $0.1<K$-factor $<10$. In this $\mathrm{K}$-factor range a small change in the fading environment has a direct impact on correlation. Once the NLOS fading environment degraded to LoS with $K$-factor $>10$, a further degradation of the NLOS environment has no effect on the correlation coefficient.

\section{B. Diversity Gain Results}

With the proposed technique the influence of the K-factor on diversity gain can also be analyzed. Fig. 6 shows the cumulative probability density function versus relative power for system B with different $\mathrm{K}$-factors. From this figure it becomes clear that the different curves with $K$-factors $<1$ cannot be differentiated, representing a typical Rayleigh-fading distribution. From K-factors between 1 and 10000, the curves approach a perpendicular line to the $x$-axis. Consequently, diversity gain decreases with increasing $\mathrm{K}$-factors, which was expected but tested in a MSC for the first time. Fig. 7 depicts the diversity gain versus $\mathrm{K}$-factor with probability level as parameter for all tested systems. In a similar way to what happened for correlation, Fig. 7 also provides for a three-stage effect of the $\mathrm{K}$-factor, this time for diversity gain. Roughly constant diversity gain values are observed for $\mathrm{K}$-factors below 0.1 . From $K=0.1$ until $K=1$ a moderate decline in the diversity gain happens. This decline becomes more pronounced for $K=1$ until $K=10$. Finally, for $K>10$ there is no diversity gain as this value approaches 0 for any given probability level and array type. Fig. 7 also offers an interesting comparison between tested systems. For a $5.8 \mathrm{~dB}$ of diversity gain and a cumulative probability of $1 \%$, the highest possible K-factor is $0.001,2$ and 3.5 for system A, B and $\mathrm{C}$ respectively. This means that system $\mathrm{C}$, with the lowest correlation but the largest volume, represents a stronger design against a degradation of the NLOS characteristics of the fading scenario. Yet, it is also observed from these figures that the inherent advantages of system types are mitigated with increasing K-factor.

For example the difference between selection combined of system $\mathrm{A}$ and $\mathrm{C}$ for $K=0.1$ and a cumulative probability of $0.2 \%$ is $9 \mathrm{~dB}$, while the difference between these same systems for $K=10$ and the same probability is only $1 \mathrm{~dB}$. This means that with $K=0.1$ the influence of the array geometry is very important, while the influence of the array geometry for $K=$ 10 onwards is very small. This could be taken into account by MIMO engineers to decide when it may be necessary to use only a selection of separated antennas or the whole receiving array as a functions of fading scenarios where the system is under operation. As a general recommendation, it can be said that for $\mathrm{K}$-factors below 10, an increase in the spacing between antennas may be useful. Also as a general rule, it can be established that for lower cumulative probability and lower K-factors, the effect of MIMO array geometry on diversity gain is more pronounced.

\section{Channel Eigenvalues}

The eigenvalues limit the capacity of the channel. Therefore, this limitation can be observed by studying their evolution with changing K-factor. Fig. 8 illustrates the mean eigenvalues for tested system B. It can be seen that the first eigenvalue is always constant regardless of the $\mathrm{K}$-factor value. Yet, the second and third eigenvalues change with changing $\mathrm{K}$-factor. It is important to note that for system B the eigenvalues do not change when $K<1$. Fig. 9 shows the eigenvalues response versus K-factor for all tested systems. From Fig. 9 it can be seen that while the first eigenvalue is practically equal in all three systems, eigenvalues 2 and 3 decrease with the same slope for all systems when $K$-factors $>1$. That is, with $K \leq 1$ the eigenvalues of the channel matrix remain constant. The eigenvalues of the channel matrix starts changing when $K>1$ for all tested systems. This indicates that the evolution of the eigenvalues depends only on the K-factor and not so strongly on the specific characteristic of the MIMO array. 


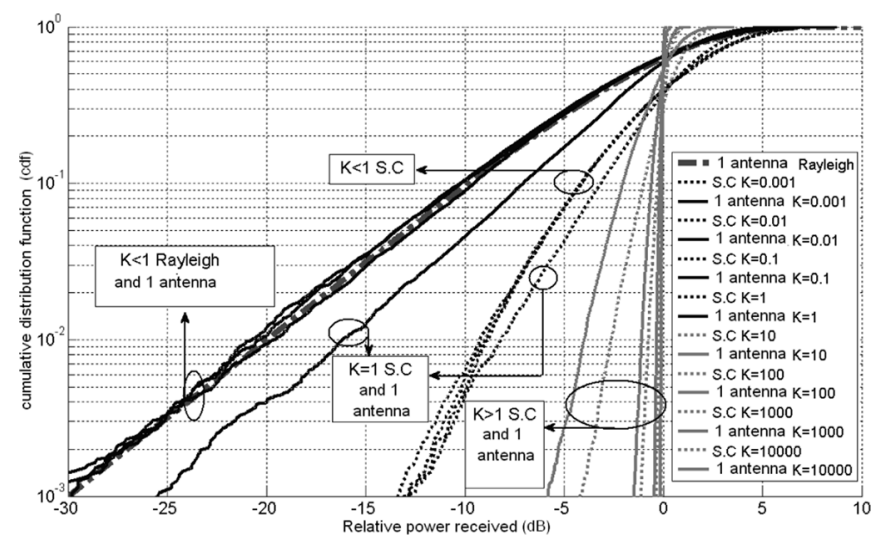

Fig. 6. Cumulative probability density function versus relative power for system B with different K-factors.

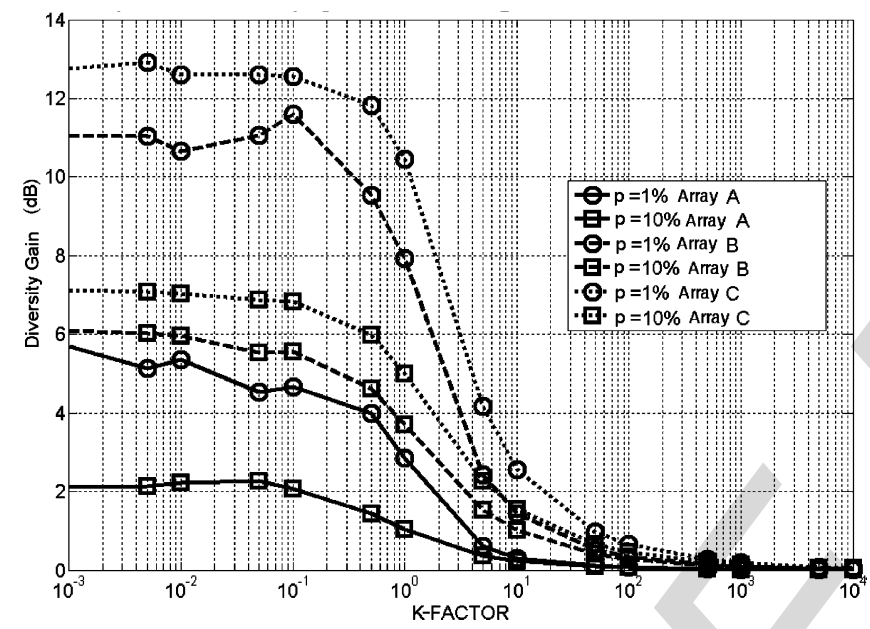

Fig. 7. Diversity gain versus K-factor with probability level as parameter, for all tested systems.

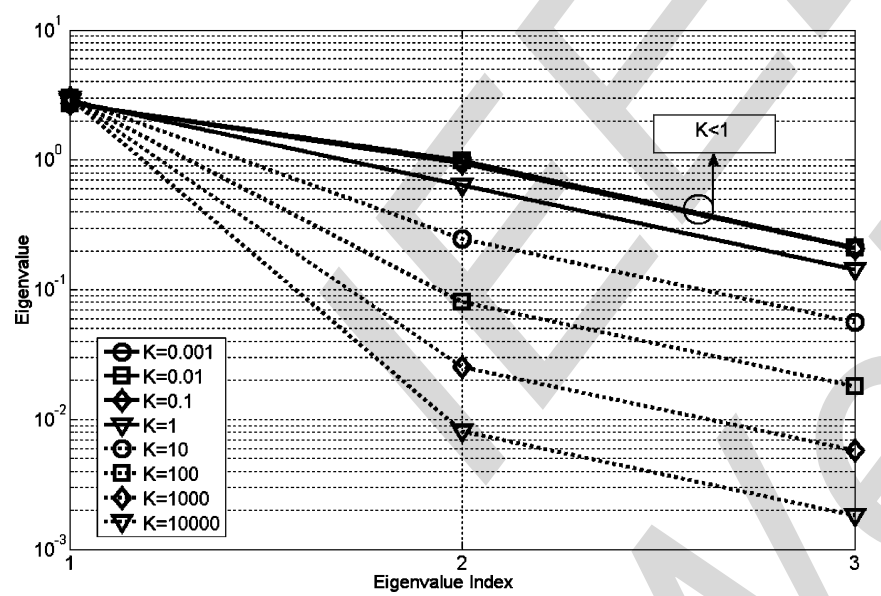

Fig. 8. Mean eigenvalues for tested system B

\section{MIMO Capacity Results}

The capacity of MIMO channels can be written as [21], [22]

$$
C_{M I M O}=\log _{2}\left(\operatorname{det}\left(I_{R}+\frac{S N R^{*}}{T} H^{*} H^{\prime}\right)\right) \frac{\frac{\text { bits }}{\mathrm{s}}}{\mathrm{Hz}}
$$

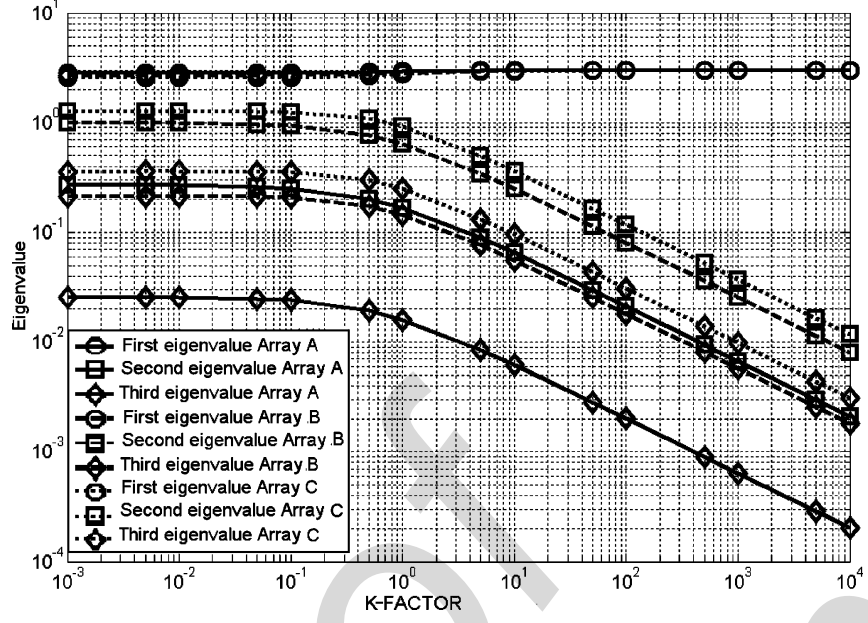

Fig. 9. Mean eigenvalues versus K-factor for all tested systems.

with $H$ the channel matrix, $T$ the number antennas at the transmitter, $R$ the number antennas at the receiver and $I_{R}$ as the identity matrix with dimension $R$. Let $\left[\lambda_{1}, \lambda_{2}, \ldots, \lambda_{\mathrm{Nmin}}\right]$ denote the nonzero eigenvalues of the $\left(N_{\min } \times N_{\min }\right)$ matrix, $H^{*} H^{\text {, }}$ with $N_{\min }=\min [T, R]$, then the capacity in (6) can be written as [23]

$$
C_{M I M O}=\sum_{i=1}^{N_{\min }} \log _{2}\left(1+\frac{S N R}{T} * \lambda_{i}\right) \frac{\frac{\text { bits }}{\mathrm{s}}}{\mathrm{Hz}} .
$$

Fig. 10 shows the measured capacity for all tested systems versus SNR for different K-factors. Fig. 11 depicts the same measured capacity versus $\mathrm{K}$-factor for different SNR values. Fig. 10 clearly shows that system capacity decreases with increasing $\mathrm{K}$-factor, as expected. The decrement is more noticeable for very high SNR values, as it was also expected. As an example, for system B and a SNR $=30 \mathrm{~dB}$, the capacity loss with regard to the case with $K=0.001,1$ and 10000 is 1.6 $\mathrm{bit} / \mathrm{s} / \mathrm{Hz}(7 \%), 6.1 \mathrm{bit} / \mathrm{s} / \mathrm{Hz}$ (26.9\%) and $11 \mathrm{bit} / \mathrm{s} / \mathrm{Hz}$ (48.7\%), respectively. For very high K-factors all systems exhibit the same capacity, as it was also expected. Similarly, the minimum SNR required for achieving a specific system capacity can be extracted from Fig. 11. For example, in order to reach a system B capacity of $10 \mathrm{bits} / \mathrm{s} / \mathrm{Hz}$, a SNR $=15,16.3,20,23.2$ and 25 would be required for K-factors of $0.001,0.1,1,10,100$ and 1000, respectively. Some examples of SNR values required to reach specific capacities at different $\mathrm{K}$-factors are listed in Table II.

For example, if a capacity greater than $15 \mathrm{bit} / \mathrm{s} / \mathrm{Hz}$ is required, this can be done with a SNR $=25 \mathrm{~dB}$ and $K<3$ or a $\mathrm{SNR}=35 \mathrm{~dB}$ and $K<350$. This requirement would not be fulfilled for SNR $<15 \mathrm{~dB}$. Finally, it is also clear from Fig. 11 that for high K-factors and given a specific SNR value, all systems achieve the same capacity. The capacity increase when changing a MIMO system to a different one out of Table I is illustrated in Table III with respect to the K-factor. From this Table it is also extracted that the change from system A to B produce a considerable capacity increase (over 15\%) for $K$-factor $\leq 50$ with SNR $=35 \mathrm{~dB}$. The same change but with 


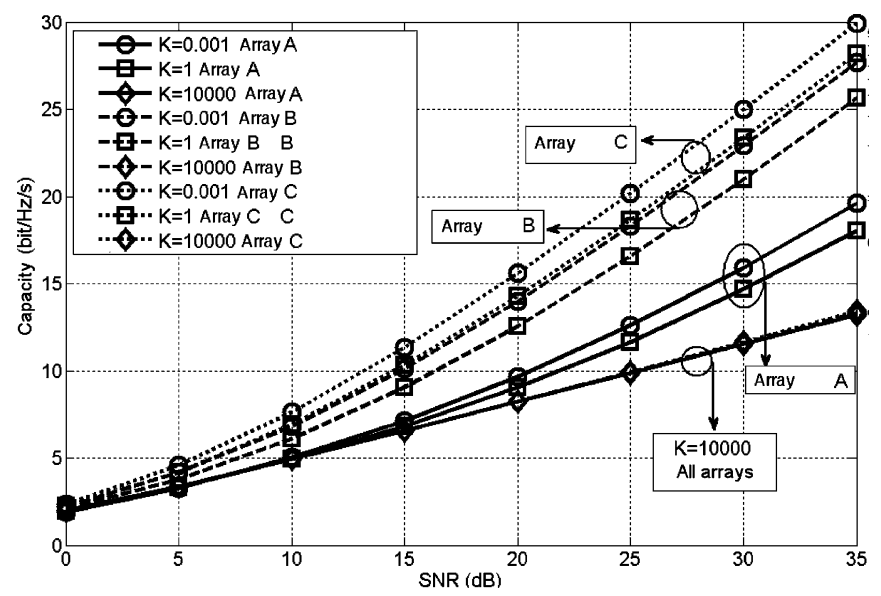

Fig. 10. Measured capacity of all tested systems versus SNR for different K-factors.

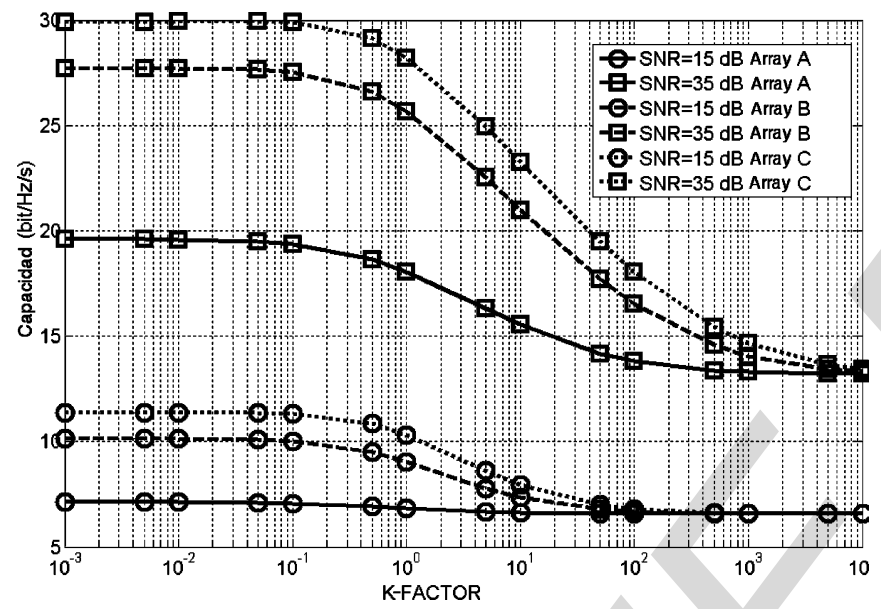

Fig. 11. Measured capacity of all tested systems versus K-factor for different SNR values.

TABLE II

REQUIRED SNR FOR DIFFERENT CAPACITIES

\begin{tabular}{|c|c|c|c|c|c|c|c|c|c|}
\hline K-factor & \multicolumn{3}{|c|}{ K=0.001 } & \multicolumn{3}{c|}{ K=1 } & \multicolumn{3}{c|}{ K=10 } \\
\hline System & A & B & C & A & B & C & A & B & C \\
\hline $\begin{array}{c}\text { Cap. } \\
\text { bits/s/H } \\
\text { z }\end{array}$ & $\begin{array}{c}\text { SNR } \\
(d B)\end{array}$ & $\begin{array}{c}\text { SNR } \\
(d B)\end{array}$ & $\begin{array}{c}\text { SNR } \\
(d B)\end{array}$ & $\begin{array}{c}\text { SNR } \\
(d B)\end{array}$ & $\begin{array}{l}\text { SNR } \\
(d B)\end{array}$ & $\begin{array}{c}\text { SNR } \\
(d B)\end{array}$ & $\begin{array}{c}\text { SNR } \\
(d B)\end{array}$ & $\begin{array}{c}\text { SNR } \\
(d B)\end{array}$ & $\begin{array}{c}\text { SNR } \\
(d B)\end{array}$ \\
\hline 10 & 20.3 & 15 & 13.4 & 22.2 & 16.1 & 14.7 & 25 & 25 & 25 \\
\hline 15 & 28.1 & 21.3 & 19.2 & 30.4 & 23.1 & 20.9 & --- & --- & --- \\
\hline 20 & ---- & 27.4 & 24.9 & ---- & 28.6 & 26.9 & --- & --- & --- \\
\hline
\end{tabular}

$\mathrm{SNR}=15 \mathrm{~dB}$, however, provides for similar capacity increments but this time for $K$-factor $<0.5$. This means that larger spatial separations in MIMO arrays provide a better benefit for high SNR scenarios.

\section{CONCLUSIONS}

Accurate emulation of any Rician-fading environment has been performed with a mode-stirred reverberation chamber for the first time. The new technique allows for greater versatility and a more detailed study of the influence of the K-factor on
TABLE III

CAPACITY INCREASE (\%) WHEN CHANGING MIMO SYSTEMS

\begin{tabular}{|c|c|c|c|c|}
\hline SNR & \multicolumn{2}{|c|}{$15 \mathrm{~dB}$} & \multicolumn{2}{c|}{$35 \mathrm{~dB}$} \\
\hline K-factor & A toB & B to C & A to B & B to C \\
\hline 0.5 & 35.7 & 13.6 & 43.0 & 10.1 \\
\hline 5 & 14.5 & 7.5 & 38.8 & 11.0 \\
\hline 50 & 2.8 & 2.8 & 25.0 & 11.4 \\
\hline 500 & 0 & 0 & 6.5 & 5.4 \\
\hline 5000 & 0 & 0 & 0.7 & 0.7 \\
\hline
\end{tabular}

MIMO performance. Correlation, diversity gain and MIMO capacity have been analyzed for three different MIMO systems under a variety of Rician-fading environments with different K-factors. Different effects have been evaluated. Results confirm previously-published influence of K-factors for outdoor measurements, yet this time with the use of a mode-stirred chamber for the first time. The proposed technique allows for a better study of K-factor influence on MIMO performance for wireless communications systems, avoiding cumbersome outdoor measurements. The results presented in this paper have been employed to extend the measuring capabilities of EMITE Ing second generation mode-stirred reverberation chambers, in addition to the emulation of non-isotropic environments. The technique represents a step forward to the goal of obtaining real-world performance from a mode-stirred reverberation chamber test. The technique is patent protected by EMITE Ing. Future research works include the hybridization of the new technique with some sample selection algorithm so as to further increase accuracy while reducing representative test samples to those important for each specific fading scenario.

\section{REFERENCES}

[1] C. Bruns and R. Vahldieck, "A closer look at reverberation chambers-3D simulation and experimental verification," IEEE Trans. Electromagn. Compat., vol. 47, pp. 612-626, Aug. 2005.

[2] K. Rosengren and P. S. Kildal, "Theoretical study of distributions of modes and plane waves in reverberation chamber for characterization of antennas in multipath environment," Microw. Opt. Technol. Lett., vol. 30, pp. 386-391, 2001.

[3] P. S. Kildal, K. Rosengren, J. Byun, and J. Lee, "Definition of effective diversity gain and how to measure it in a reverberation chamber," Microw. Opt. Technol. Lett., vol. 34, no. 1, pp. 56-59, Jul. 2002.

[4] P. S. Kildal and K. Rosengren, "Correlation and capacity of MIMO systems and mutual coupling, radiation efficiency, and diversity gain of their antennas: Simulations and measurement in a reverberation chamber," IEEE Commun. Mag., pp. 104-112, Dec. 2004.

[5] L. Greenstein, S. Ghassemzadeh, V. Erceg, and D. G. Michelson, "Theory, experiments, and statistical models," presented at the WPMC'99 Conf., Amsterdam, Sep. 1999.

[6] E. Green, "Radio link design for microcellular systems," BT Tech. J., vol. 8, no. 1, pp. 85-96, 1990.

[7] V. R. Anreddy and M. A. Ingram, "Capacity of measured Ricean and Rayleigh indoor MIMO channels at $2.4 \mathrm{GHz}$ with polarization and spatial diversity," in Proc. IEEE Wireless Communications and Networking Conf. (WCNC'06), Apr. 2006, vol. 2, pp. 946-951.

[8] J. F. Valenzuela-Valdés, A. M. Martínez-González, and D. A. SánchezHernández, "Emulation of MIMO non-isotropic fading environments with reverberation chambers," IEEE Antennas Wireless Propag. Lett., vol. 7, pp. 325-328, 2008.

[9] Z. Yun and M. F. Iskander, "MIMO capacity for realistic wireless communications environments," in Proc. IEEE Antennas and Propagation Society Int. Symp., Jun. 2004, pp. 1231-1234.

[10] P. Hallbjorner and K. Madsen, "Terminal antenna diversity characterization using mode stirred chamber," Electron. Lett., vol. 37, no. 5, pp. 273-274, Mar. 2001. 
[11] J. F. Valenzuela-Valdés et al., "Evaluation of true polarization diversity for MIMO systems," IEEE Trans. Antennas Propag., vol. 57, pp. 2746-2755, Sep. 2009.

[12] J. D. Sánchez-Heredia, M. Grudén, J. F. Valenzuela-Valdés, and D. A. Sánchez-Hernández, "Sample selection method for arbitrary fading emulation using mode-stirred chambers," IEEE Antennas and Wireless Propag. Lett., 2010, to be published.

[13] C. L. Holloway, D. A. Hill, J. M. Ladbury, P. F. Wilson, G. Koepke, and J. Coder, "On the use of reverberation chambers to simulate a Rician radio environment for the testing of wireless devices," IEEE Trans. Antennas Propag., vol. 54, pp. 3167-3177, Nov. 2006.

[14] M. Kang and M. S. Alouini, "Capacity of MIMO Rician channels," IEEE Trans. Wireless Commun., vol. 5, pp. 112-122, Jan. 2006.

[15] C. Chen-Nee, J. M. Kahn, and D. Tse, "Capacity of multi-antenna array systems in indoor wireless environment," in Proc. IEEE Global Telecommunications Conf. GLOBECOM 98, 1998, vol. 4, pp. 1894-1899.

[16] D. Chizhik, G. J. Foschini, and R. A. Valenzuela, "Capacities of multi-element transmit and receive antennas: Correlations and keyholes," Electron. Lett., vol. 36, no. 13, pp. 1099-1100, Jun. 2000.

[17] K. I. Pedersen, J. B. Andersen, J. P. Kermoal, and P. Mogensen, "A stochastic multiple-input-multiple-output radio channel model for evaluation of space-time coding algorithms," in Pro. IEEE Vehicular Technology Conf., Sep. 2000, vol. 2, pp. 893-897.

[18] J. P. Kermoal, L. Schumacher, K. I. Pedersen, P. E. Mogensen, and F. Frederiksen, "A stochastic MIMO radio channel model with experimental validation," IEEE J. Sel. Areas Commun., vol. 20, pp. 1211-1226, Aug. 2002.

[19] D. Chizhik, J. Ling, P. W. Wolniansky, R. A. Valenzuela, N. Costa, and K. Huber, "Multiple-input-Multiple-output measurements and modelling in Manhattan," IEEE J. Sel. Areas Commun., vol. 21, pp. 321-331, 2003.

[20] W. Weichselberger, M. Herdin, H. Ozcelik, and E. Bonek, "A stochastic MIMO channel model with joint correlation of both link ends," IEEE Trans. Wireless Commun., vol. 5, pp. 90-100, Jan. 2006.

[21] G. Foschini and M. Gans, "On limits of wireless communication in a fading environment when using multiple antennas," Wireless Personal Commun., pp. 311-335, Mar. 1998.

[22] E. Telatar, "Capacity of multi-antenna Gaussian channels," Eur. Trans. Telecommun., vol. 10, no. 6, pp. 585-595, Nov./Dec. 1999.

[23] J. Winters, "On the capacity of radio communication systems with diversity in a Rayleigh fading environment," IEEE J. Sel. Areas Commun., vol. 5, pp. 871-878, Jun. 1987.

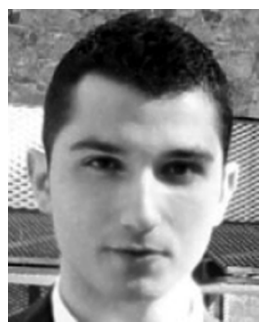

Juan D. Sánchez-Heredia was born in Lorca, Spain. He received the Telecommunication Engineering Degree from the Universidad Politécnica de Cartagena, Spain, in 2009, and is working toward the Masters degree in information technologies at the Universidad de Murcia, Spain.

In 2007, he worked at General Electric (Cartagena), and was involved in several projects in relation with the network infrastructure. In 2009, he joined the Department of Information Technologies and Communications, Universidad Politécnica de Cartagena, Spain. His current research areas cover MIMO communications, multimode-stirred chambers and electromagnetic dosimetry.

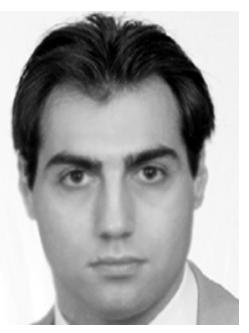

Juan F. Valenzuela-Valdés was born in Marbella, Spain. He received the Degree in Telecommunications Engineering from the Universidad de Malaga, Spain, in 2003 and the Ph.D. from Universidad Politécnica de Cartagena, in May 2008.

In 2004, he worked at CETECOM (Malaga). In 2004, he joined the Department of Information Technologies and Communications, Universidad Politécnica de Cartagena, Spain. In 2007, he joined EMITE Ing as Head of Research. His current research areas cover MIMO communications, multimode-stirred chambers and SAR measurements.

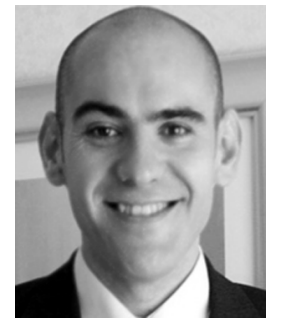

Antonio M. Martínez-González received the Dipl.-Ing. degree in telecommunications engineering from the Universidad Politécnica de Valencia, Spain, in 1998 and the Ph.D. degree from the Universidad Politécnica de Cartagena, Spain, in 2004

From 1998 until September 1999, he was employed as a Technical Engineer at the Electromagnetic Compatibility Laboratory, Universidad Politécnica de Valencia, where he developed assessment activities and compliance certifications with European directives related with immunity and emissions to electromagnetic radiation from diverse electrical, electronic and telecommunication equipment. Since September 1999, he has been an Associate Professor at the Universidad Politécnica de Cartagena. At present, his research interest is focused on electromagnetic dosimetry, radioelectric emissions and mode stirred chambers applied to MIMO systems. In December 2006, he was one of the founders of EMITE Ing, a technological spin-off company founded by telecommunication engineers and doctors of the Microwave, Radiocommunications and Electromagnetism Research Group (GIMRE), Technical University of Cartagena (Spain).

Prof. Martínez-González was awarded with the Spanish National Prize from Foundation Airtel and Colegio Oficial de Ingenieros de Telecomunicación de España for the best final project on mobile communications in 1999. In 2006 and 2008, the second i-patentes prize for innovation and technology transfer in the Region of Murcia (Spain) was awarded to the founders of EMITE Ing.

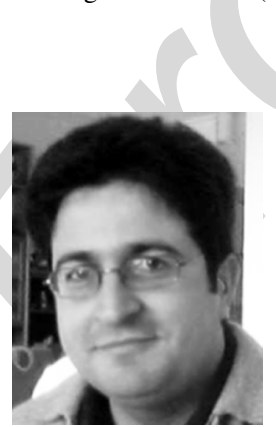

David A. Sánchez-Hernández (M'00-SM'06) received the Dipl.-Ing. degree in telecommunications engineering from the Universidad Politécnica de Valencia, Spain, in 1992 and the Ph.D. degree from King's College, University of London, London, U.K., in early 1996.

From 1992 to 1994 , he was employed as a Research Associate for The British Council-CAM, King's College London, where he worked on active and dual-band microstrip patch antennas. In 1994, he was appointed EU Research Fellow at King's College London, working on several joint projects at 18,38 and $60 \mathrm{GHz}$ related to printed and integrated antennas on GaAs, microstrip antenna arrays, sectorization and diversity. In 1997, he returned to Universidad Politécnica de Valencia, Spain, where was co-leader of the Antennas, Microwaves and Radar Research Group and the Microwave Heating Group. In early 1999, he received the Readership from Universidad Politécnica de Cartagena, and was appointed Vice Dean of the School for Telecommunications Engineering and Leader of the Microwave, Radiocommunications and Electromagnetism Engineering Research Group. In late 1999, he was appointed Vice Chancellor for Innovation \& Technology Transfer at the Universidad Politécnica de Cartagena and a member of several Foundations and Societies for promotion of R\&D in the Autonomous Region of Murcia, in Spain. In May 2001, he was appointed official advisor in technology transfer and a member of The Industrial Advisory Council of the Autonomous Government of the Region of Murcia, Spain. In May 2003 he was appointed Head of Department and, in June 2009, he received the Chair at the Universidad Politécnica de Cartagena, Spain. He has published three international books, over 45 scientific papers and over 90 conference contributions, and is a reviewer of several international journals. He holds six patents. His current research interests encompass all aspects of the design and application of printed multi-band antennas for mobile communications, electromagnetic dosimetry issues and MIMO techniques for wireless communications.

Dr. Sánchez-Hernández is a Chartered Engineer (CEng), IET Fellow, IEEE Senior Member, CENELEC TC106X member, and is the recipient of the R\&D J. Langham Thompson Premium, awarded by the Institution of Engineering and Technology, the i-Patentes award to innovation and technology transfer, the Emprendedor XXI award to innovative entrepreneurship, granted by the Spanish National Innovation Entity (ENISA), as well as other national and international awards. 


\title{
Emulation of MIMO Rician-Fading Environments With Mode-Stirred Reverberation Chambers
}

\author{
Juan D. Sánchez-Heredia, Juan F. Valenzuela-Valdés, Antonio M. Martínez-González, and \\ David A. Sánchez-Hernández, Senior Member, IEEE
}

\begin{abstract}
Some recent publications have extended the emulating capabilities of mode-stirred reverberation chambers, which are now able to emulate Ricean-fading and non-isotropic environments. Either the need to physically modify existing chambers or multiple sets of measurements is required for these enhancements. In this paper a novel post-processing hybrid tool is presented for the transformation of a Rayleigh-fading emulated environment into a Rician one. The model is tested and compared to outdoor measurements and simulations through the K-factor, correlation, diversity gain and MIMO capacity. Results show an excellent matching performance with no hardware modifications of mode-stirred chambers with just one set of measurements. The method is patent protected by EMITE Ing.
\end{abstract}

Index Terms-Channel capacity, diversity gain, multiple-inputmultiple-output (MIMO) systems, radiation efficiency.

\section{INTRODUCTION}

A single-cavity mode-stirred reverberation chamber, also known as a reverberation chamber (RC), is a metal cavity sufficiently large to support many resonant modes. Although RCs have been extensively used in electromagnetic compatibility problems [1], their use for antennas and propagation-related problems have only acquired attention in the last few years. This is due to their ability to artificially generate a repeatable multipath environment. RCs were initially thought to provide an isotropic and randomly polarized measuring scenario. This was done by perturbing the modes with stirrers and rotating platforms. In this way a fading environment similar to the ones found in indoor and urban environments (Rayleigh) but with a uniform elevation distribution of the incoming waves is obtained [2], [3]. The real and imaginary parts of the received signal become then normally (Gaussian) distributed, with the associated magnitude following a Rayleigh distribution and the phase following a uniform distribution over

Manuscript received October 26, 2009; revised June 16, 2010; accepted October 25, 2010. Date of publication December 03, 2010; date of current version February 02, 2011. This work was supported in part by Fundación Séneca, the R\&D unit of the Autonomous Region of Murcia (Spain) under project reference 11783/PI/09 and in part by the Spanish National R\&D Programme through TEC2008-05811.

J. Valenzuela-Valdés is with the EMITE Ingeniería SLNE, Edificio CEEIM, E-30100 Espinardo, Murcia, Spain (e-mail: juan.valenzuela@emite-ingenieria. es).

J. D. Sánchez-Heredia, A. M. Martínez-González, and D. A. SánchezHernández are with the Departamento de Tecnologías de la Información y Comunicaciones, Universidad Politécnica de Cartagena, Cartagena E-30202, Spain (e-mail: david.sanchez@upct.es).

Color versions of one or more of the figures in this paper are available online at http://ieeexplore.ieee.org.

Digital Object Identifier 10.1109/TAP.2010.2096185
$2 \pi$. While avoiding cumbersome outdoor measurements, compact-size RCs provide accurate, repeatable and reliable ways of validating measurements for antenna systems and diversity schemes [4]. Yet, real propagating scenarios rarely follow an ideal Rayleigh-fading environment. The K-factor of more general Rician-fading environments changes as the distance of receiver to transmitter changes [5]. Macrocells usually offer a greater K-factor than microcells [6] while rich multipath environments provide $\mathrm{K}$-factors typically close to 0 [7]. Consequently, recent research efforts have concentrated on extending the original capabilities of first-generation RCs. Good examples are the recent extension to non-isotropic environments [8] and the emulation of the effects of metallic windows and other artefacts, trees and walls in buildings [9], although this last one only for Rayleigh-fading scenarios. Unlike reverberation chambers (RCs), where only one cavity is used, MSCs may contain more than one metal cavity. Cavities are coupled by diverse means and a rich fading environment consisting on diverse clusters with different fading characteristics can be obtained. The MSC also contains a set of mode stirrers that change the boundary conditions of the main cavity within the chamber. This causes a multi-reflective environment which is repeatable and can be statistically studied. MSCs can be used as a very fast, easy, and accurate tool to measure a wide variety of MIMO parameters for different extension of the performance of conventional RCs [10]-[12]. With the use of appropriate equipment and through adequate processing of the measured scattering (S) parameters, radiation efficiency, self-impedance, total radiated power, total isotropic sensitivity and several MIMO channel performance variables, including bit error rate (BER), can now be evaluated using MSCs. The extension to emulate Rician-fading [13] has also been available recently. In this Rician-fading emulation technique, however, either complex hardware alterations or large sets of measurements are required. In [13], the Rician-fading environment is obtained at the cost of varying the chamber characteristics and/or the antenna configuration. There is an inherently higher hardware complexity to this option.

In this paper, a novel mode-processing technique which allows for an emulation of Rician-fading environments from the data taken in a mode-stirred reverberation chamber with Rayleigh-fading emulated environment is presented. The method is not intended to describe in detail the response of a device to a change in the angle of arrival (AoA), but rather uses a deterministic component of a Rician signal via post processing to observe the associated changes in the conventional post-processing techniques used to determine general MIMO parameters of the device, such as diversity gain and MIMO 


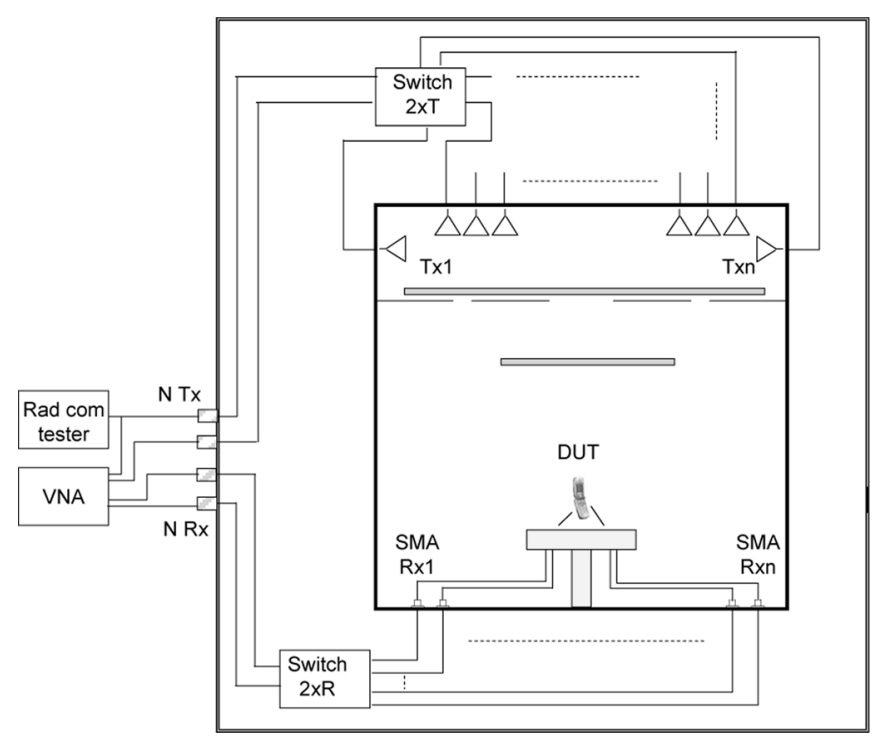

Fig. 1. Sketch of measurements with the E200 MIMO analyzer.

capacity. The method allows the MIMO designer to evaluate several Rician K-factors environments with a single measurement set in the MSC. With this technique a considerable reduction of chamber cost and complexity as well as measuring time is provided, alleviating the hardware complexity of other techniques. Section II describes the proposed measurement and emulation technique for Rician-fading environments. The method is validated through a comparison to other simulated and measured results. Channel eigenvalues, correlation, diversity gain and system capacity results for several scenarios are described in Section III. Finally, some conclusions are outlined in Section IV.

\section{Emulating AND MEAsuring Technique}

Measurements illustrated in this paper have been performed with the EMITE Ing $8 \times 8$ MIMO Analyzer Series E200a in connection to the Rohde \& Schwarz ZVRE Vector Network Analyzer $(9 \mathrm{kHz}$ to $4 \mathrm{GHz})$. The MIMO Analyzer is a second generation two-cavity mode-stirred reverberation chamber with external dimensions of $0.82 \mathrm{~m} \times 1.275 \mathrm{~m} \times 1.95 \mathrm{~m}, 8$ exciting antennas, polarization stirring due to aperture-coupling and to the different orientation of the antenna exciting elements, 3 mechanical and mode-coupling stirrers, 1 holder-stirrer and variable iris-coupling. The MIMO Analyzer was set-up for 3 holder positions with 15 different mechanical stirrer positions for each holder position, 12 iris-coupling aperture stirring and $20 \mathrm{MHz}$ frequency stirring. Measurements were performed at $1800 \mathrm{MHz}$ and half-wave dipoles were used as MIMO antennas. A sketch of the measurement setup can be observed from Fig. 1.

The K-factor, defined as the ratio of the direct path component to the scattered component can be calculated from measured S-parameter in a mode-stirred reverberation chamber by [13],

$$
K=\frac{\text { direct component }}{\text { scatterd components }}=\frac{d_{R}^{2}}{2 \sigma_{R}^{2}}=\frac{\left(\left|\left\langle s_{21}\right\rangle\right|\right)^{2}}{\left\langle\left|s_{21}-\left\langle s_{21}\right\rangle\right|^{2}\right\rangle}
$$

where $S_{21}$ is the measured parameter in the mode-stirred reverberation chamber for each antenna pair, $\sigma_{R}$ is the radius of cluster data and $d_{R}$ is the distance of centroid of cluster from the origin. A scatter plot would result in the data clustered in a circle and centered about the origin for pure Rayleigh-fading environments $(K=0)$. As the direct line of sight (LoS) energy became comparable to the non-LoS energy, the cluster of data would move away from the origin, and the fading environment becomes a Rician one. Scatter plots are useful not only for identifying the LoS behavior of the fading environments, but also to quantify the data dispersion through the K-factor. The data set measured with the chamber has to be large enough to ensure a Rayleigh-fading scenario. While this is a typical use of RCs, it also means that both the direct and non-direct coupling paths are included in the data set in a more complex mode-coupling muticavity MSC. It is clear from (1) that by increasing the direct component the $\mathrm{K}$-factor will increase. Consequently, we can move the cluster data away from the origin by adding an offset continuous component. The offset has to be a complex number by

$$
\begin{array}{r}
\text { off set }=\mid \text { offset }\left.\right|^{*} \cos \left(\text { off } \text { set }_{\varangle}\right) \\
+i^{*} \mid \text { off set }\left.\right|^{*} \operatorname{sen}\left(\text { off set } t_{\varangle}\right) .
\end{array}
$$

For a target K-factor $\left(K_{\text {target }}\right)$, the required offset can be defined by

$$
\mid \text { off set } \mid=\sqrt{K_{\text {target }}{ }^{*} 2 \sigma_{R}^{2}-\left|d_{R}\right|} .
$$

The phase of the offset in (3) is obtained from the averaged phase of all data samples in the initial set. Equation (3) can be re-written in terms of the S-parameters by

$$
\mid \text { off set }\left|=\sqrt{K_{\text {target }}{ }^{*} 2\left\langle\left|s_{21}-\left\langle s_{21}\right\rangle\right|^{2}\right\rangle}-\right|\left\langle s_{21}\right\rangle \mid .
$$

In order to obtain the desired results, the added offset would have to be phase-coherent to the selected radius in the way

$$
\text { off set }_{\varangle}=d_{R \varangle}=\left\langle s_{21}\right\rangle_{\varangle} .
$$

The calculated offset has to be added to all samples that are used for the Rician emulation. In this way the new Rician-fading samples keep the standard deviation of the original Rayleighfading ones, and therefore the distance can be altered to be adapted to the target K-factor. Fig. 2 depicts several scatter plots for the original Rayleigh-fading samples and different Ricianfading ones with $K=5,15$ and 100 using the proposed technique.

It is clearly observed from this figure that the modified samples keep the standard deviation while their radius varies in a proportional way to the target K-factor. Fig. 3 shows a comparison between measured histograms for diverse sample sets with their associated probability distribution functions and several emulated sample sets. Originally-emulated samples can clearly be associated to a Rayleigh-fading scenario, while modified samples conform very well to measured Rician-fading 


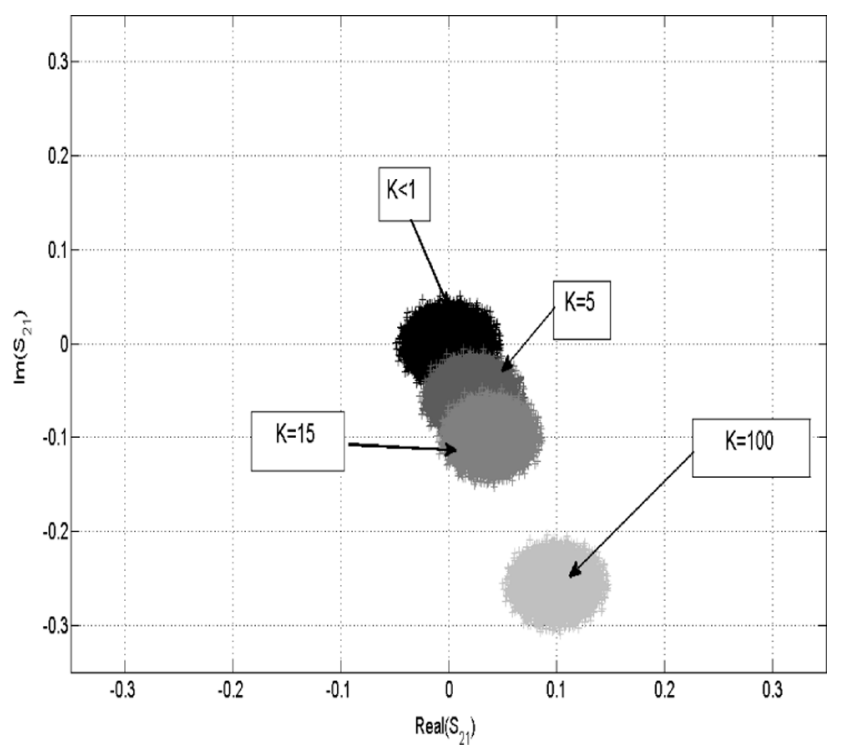

Fig. 2. Scatter plots for mode-stirred and mode-processed data.

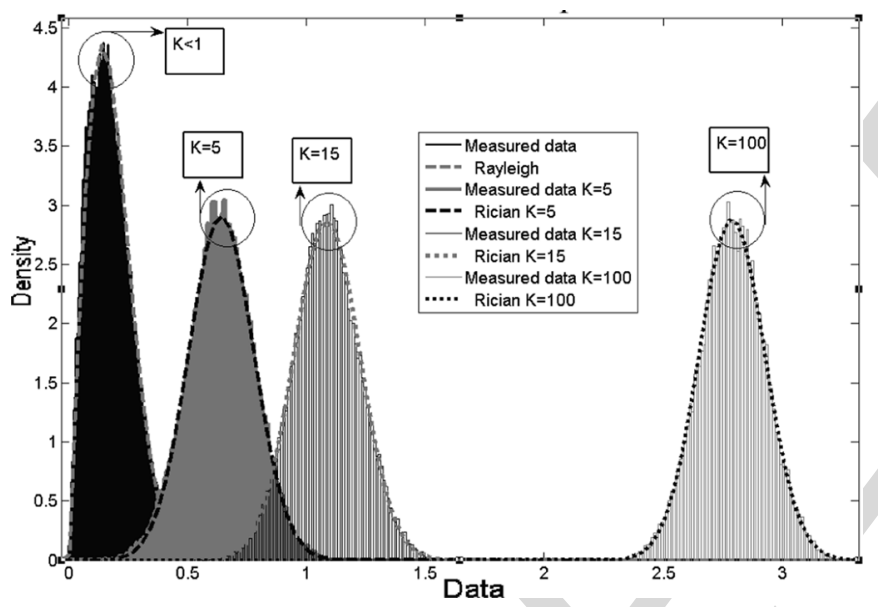

Fig. 3. Histograms for measured and modified data.

scenarios with $K=5,15$ and 100. It seems clear, however, that the method relies on the previous estimation of the initial $K$ factor of the sample set. Consequently, the uncertainties of this method are linked to the uncertainties of the method employed to estimate the initial $K$ factor of the sample set.

In order to validate the proposed technique, 3 different initial $3 \times 3$ MIMO systems were measured. Three $\lambda / 2$ dipoles in a vertical position were employed as antennas in reception. The difference between the tested arrays as the spatial separation between the receiving dipoles, as shown in Table I. The initial measured K-factor was always below 0.0001 for the 3 systems, that is, clearly a Rayleigh-fading environment. The initial K-factors were then incremented slightly to between $10^{-3}$ and $10^{3}$ using the proposed technique. In this way, with only 3 system measurements in a Rayleigh-fading environment, it was possible to emulate and evaluate their performance for any Rician-fading environment. This drastically reduces both R\&D costs and time. The emulated results were compared with the outdoor measurements results in [14]. This comparison is shown

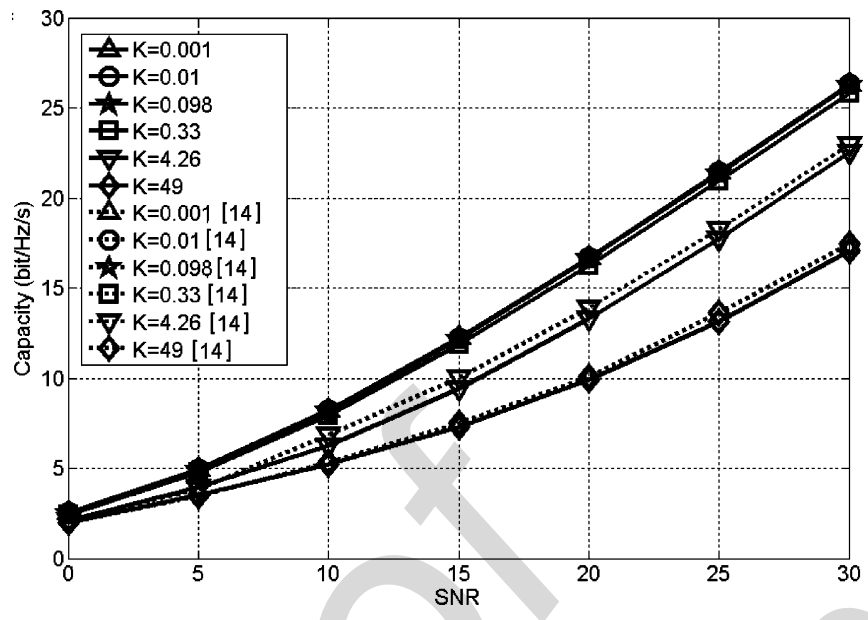

Fig. 4. Measured and emulated capacity versus SNR for $3 \times 3$ MIMO systems

TABLE I

MEASURED MIMO ARRAYS

\begin{tabular}{|c|c|c|}
\hline System & $\begin{array}{c}\text { Spatial separation between } \\
\text { adjacent antennas }(\mathrm{d} / \lambda)\end{array}$ & $\begin{array}{c}\text { Initial measured K- } \\
\text { factor }\end{array}$ \\
\hline $\mathrm{A}$ & 0.01 & $<0.0001$ \\
\hline $\mathrm{B}$ & 0.05 & $<0.0001$ \\
\hline $\mathrm{C}$ & 0.10 & $<0.0001$ \\
\hline
\end{tabular}

in Fig. 4. Good agreement is observed between outdoor measurements and measurements emulated with the proposed technique. Thus, this method does not intend to describe the specific response of the device to a change in the specific angle of arrival (AoA) characteristic of a fading scenario, but rather to describe the changes in the general performance of the device when the scenario contains an increased LoS component. In Fig. 4, the method closely follows the real evaluation of a device for changing $\mathrm{K}$ factors in outdoor measurements made in [14]. This is clear evidence that the technique represents a step forward to the goal of obtaining real-world performance from a mode-stirred chamber test.

\section{MEASURED RESUlts}

\section{A. Correlation Results}

A traditional parameter to be analyzed is the correlation between antennas, which gives us an idea of signal similarities. This parameter has been postulated as sufficient to characterize the MIMO systems in some popular models such as the Kronecker model [15]-[18]. Recent analyses, however, have raised the need of more information to accurately predict system capacity [19], [20]. With the aid of the proposed technique, the correlation properties in terms of the fading environment could be studied. In this way we can analyze how does the correlation coefficient change from Rayleigh-fading environments $(K=0)$ as $\mathrm{K}$-factors are increased. Fig. 5 shows the correlation coefficient between adjacent antennas for the different systems in Table I. From this figure one can observe that different correlation characteristics exist for the different systems. As it was expected, the system with highest correlation coefficient is system 


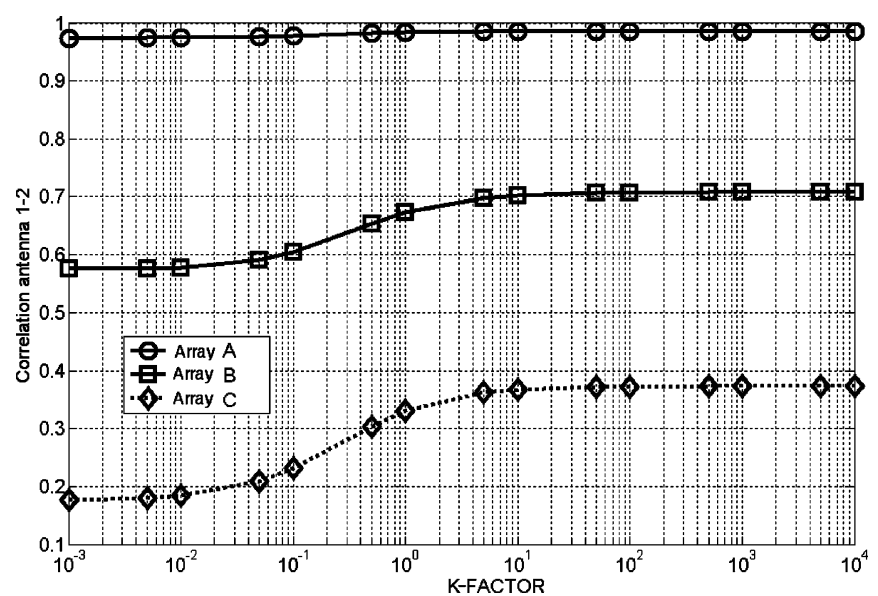

Fig. 5. Emulated correlation coefficients between adjacent antennas for the MIMO systems in Table I.

A. This is because of its smallest separation distance between adjacent vertically-polarized dipole antennas, which leads to the highest correlation coefficient. In consequence, system $\mathrm{C}$ depicts the lowest correlation coefficients. It is also important to note from Fig. 5 that the correlation coefficients change with changes in the $\mathrm{K}$-factor. While the increment of correlation in system A with increasing $\mathrm{K}$ factor is almost imperceptible $(0.97$ to 0.98 ), system $C$ nearly doubles this parameter (0.18 to 0.39 ), while system B provides for a $22.4 \%$ increment $(0.58$ to 0.71$)$. Therefore, one can conclude that when increasing the K-factor the correlation coefficient also increases, and this increment is more noticeable when the correlation factor is low. In other words, the influence of the K-factor is more important for systems with low correlation between adjacent antennas. It is also important to stress that the increase in the change in the correlation coefficient that occurs when varying the $\mathrm{K}$-factor has a limitation. For K-factors below 0.01 the correlation remains constant. For K-factors between 0.01 and 0.1 the correlation increases very quickly with increasing K-factor. This K-factor range accounts for $10 \%$ of the total correlation increment. For $\mathrm{K}$-factors between 0.1 and 10 , the correlation increment slope is more pronounced, achieving in this $\mathrm{K}$-factor range a $90 \%$ of the total increase in correlation. Finally, for K-factors above 10 the correlation coefficient again remains constant. This means that the environment influences the correlation factor in a specific K-factor range. In other words, the K-factor plays a role on correlation coefficients for $0.1<K$-factor $<10$. In this $\mathrm{K}$-factor range a small change in the fading environment has a direct impact on correlation. Once the NLOS fading environment degraded to LoS with $K$-factor $>10$, a further degradation of the NLOS environment has no effect on the correlation coefficient.

\section{B. Diversity Gain Results}

With the proposed technique the influence of the K-factor on diversity gain can also be analyzed. Fig. 6 shows the cumulative probability density function versus relative power for system B with different $\mathrm{K}$-factors. From this figure it becomes clear that the different curves with $K$-factors $<1$ cannot be differentiated, representing a typical Rayleigh-fading distribution. From K-factors between 1 and 10000, the curves approach a perpendicular line to the $x$-axis. Consequently, diversity gain decreases with increasing $\mathrm{K}$-factors, which was expected but tested in a MSC for the first time. Fig. 7 depicts the diversity gain versus $\mathrm{K}$-factor with probability level as parameter for all tested systems. In a similar way to what happened for correlation, Fig. 7 also provides for a three-stage effect of the $\mathrm{K}$-factor, this time for diversity gain. Roughly constant diversity gain values are observed for $\mathrm{K}$-factors below 0.1 . From $K=0.1$ until $K=1$ a moderate decline in the diversity gain happens. This decline becomes more pronounced for $K=1$ until $K=10$. Finally, for $K>10$ there is no diversity gain as this value approaches 0 for any given probability level and array type. Fig. 7 also offers an interesting comparison between tested systems. For a $5.8 \mathrm{~dB}$ of diversity gain and a cumulative probability of $1 \%$, the highest possible K-factor is $0.001,2$ and 3.5 for system A, B and $\mathrm{C}$ respectively. This means that system $\mathrm{C}$, with the lowest correlation but the largest volume, represents a stronger design against a degradation of the NLOS characteristics of the fading scenario. Yet, it is also observed from these figures that the inherent advantages of system types are mitigated with increasing K-factor.

For example the difference between selection combined of system $\mathrm{A}$ and $\mathrm{C}$ for $K=0.1$ and a cumulative probability of $0.2 \%$ is $9 \mathrm{~dB}$, while the difference between these same systems for $K=10$ and the same probability is only $1 \mathrm{~dB}$. This means that with $K=0.1$ the influence of the array geometry is very important, while the influence of the array geometry for $K=$ 10 onwards is very small. This could be taken into account by MIMO engineers to decide when it may be necessary to use only a selection of separated antennas or the whole receiving array as a functions of fading scenarios where the system is under operation. As a general recommendation, it can be said that for $\mathrm{K}$-factors below 10, an increase in the spacing between antennas may be useful. Also as a general rule, it can be established that for lower cumulative probability and lower K-factors, the effect of MIMO array geometry on diversity gain is more pronounced.

\section{Channel Eigenvalues}

The eigenvalues limit the capacity of the channel. Therefore, this limitation can be observed by studying their evolution with changing K-factor. Fig. 8 illustrates the mean eigenvalues for tested system B. It can be seen that the first eigenvalue is always constant regardless of the $\mathrm{K}$-factor value. Yet, the second and third eigenvalues change with changing $\mathrm{K}$-factor. It is important to note that for system $B$ the eigenvalues do not change when $K<1$. Fig. 9 shows the eigenvalues response versus K-factor for all tested systems. From Fig. 9 it can be seen that while the first eigenvalue is practically equal in all three systems, eigenvalues 2 and 3 decrease with the same slope for all systems when $K$-factors $>1$. That is, with $K \leq 1$ the eigenvalues of the channel matrix remain constant. The eigenvalues of the channel matrix starts changing when $K>1$ for all tested systems. This indicates that the evolution of the eigenvalues depends only on the K-factor and not so strongly on the specific characteristic of the MIMO array. 


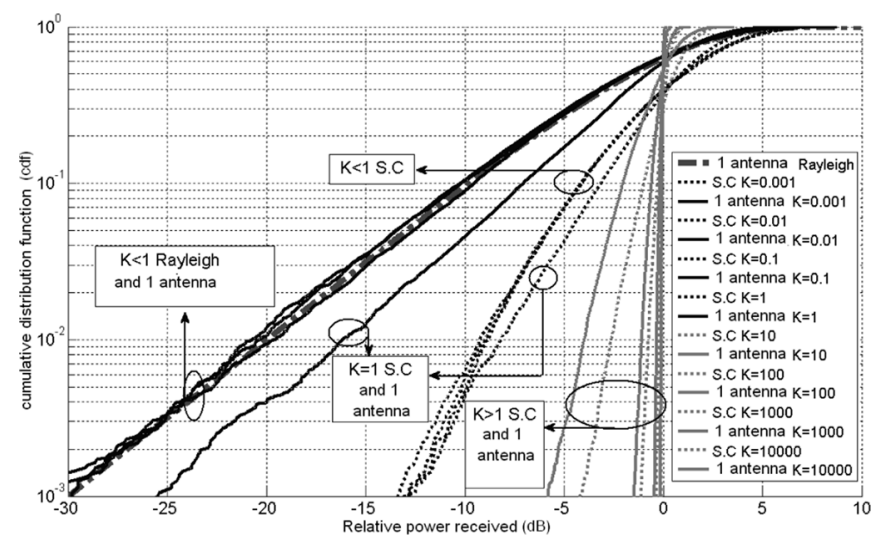

Fig. 6. Cumulative probability density function versus relative power for system B with different K-factors.

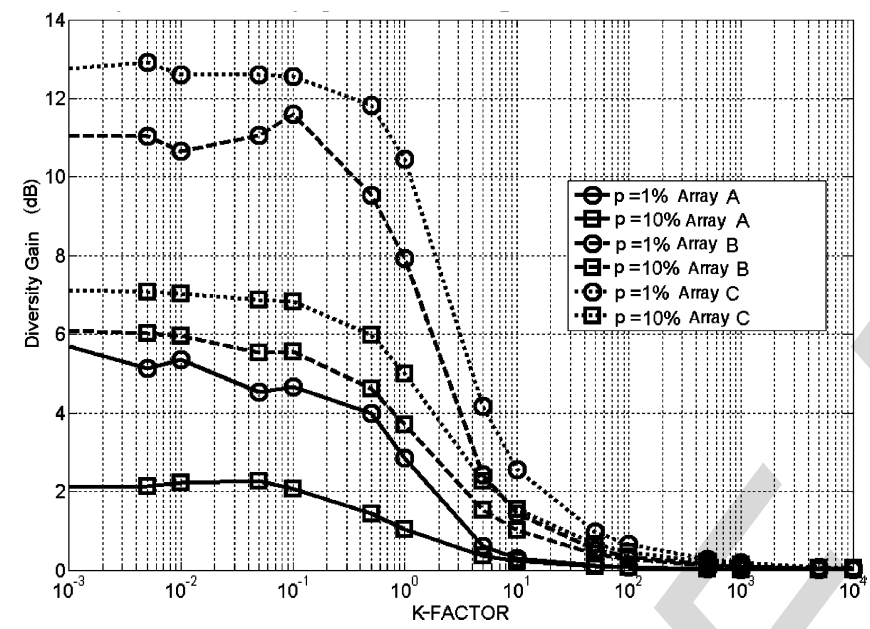

Fig. 7. Diversity gain versus K-factor with probability level as parameter, for all tested systems.

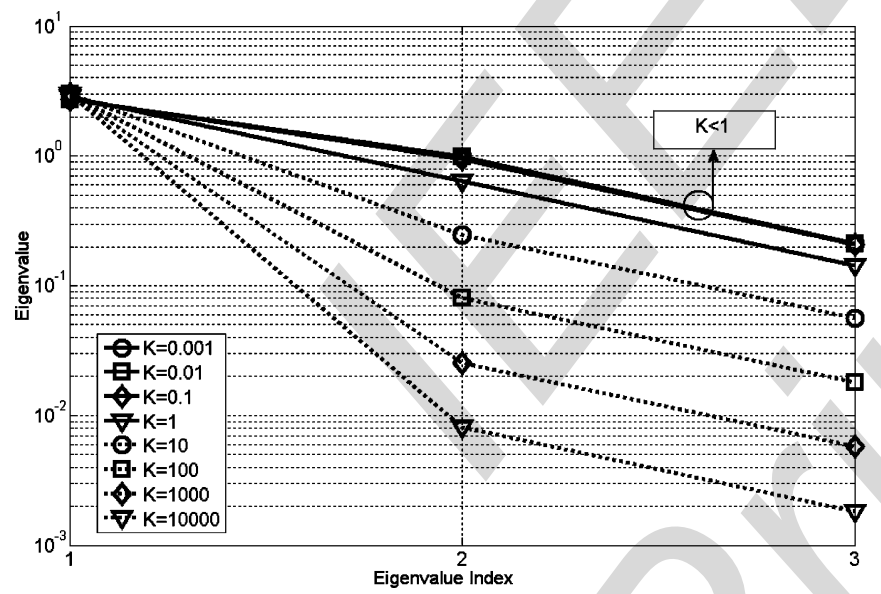

Fig. 8. Mean eigenvalues for tested system B.

\section{MIMO Capacity Results}

The capacity of MIMO channels can be written as [21], [22]

$$
C_{M I M O}=\log _{2}\left(\operatorname{det}\left(I_{R}+\frac{S N R^{*}}{T} H^{*} H^{\prime}\right)\right) \frac{\frac{\text { bits }}{\mathrm{s}}}{\mathrm{Hz}}
$$

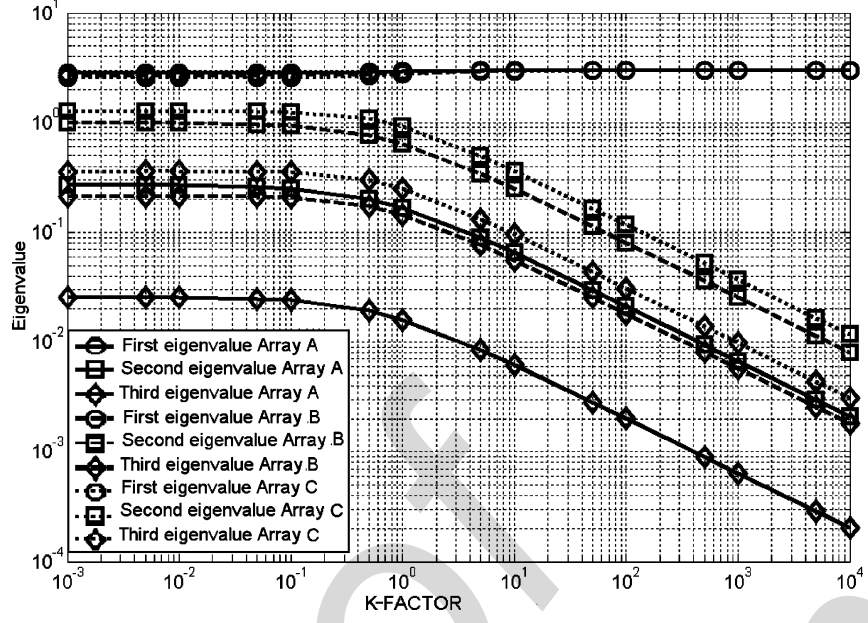

Fig. 9. Mean eigenvalues versus K-factor for all tested systems.

with $H$ the channel matrix, $T$ the number antennas at the transmitter, $R$ the number antennas at the receiver and $I_{R}$ as the identity matrix with dimension $R$. Let $\left[\lambda_{1}, \lambda_{2}, \ldots, \lambda_{\mathrm{Nmin}}\right]$ denote the nonzero eigenvalues of the $\left(N_{\min } \times N_{\min }\right)$ matrix, $H^{*} H^{\text {, }}$ with $N_{\min }=\min [T, R]$, then the capacity in (6) can be written as [23]

$$
C_{M I M O}=\sum_{i=1}^{N_{\min }} \log _{2}\left(1+\frac{S N R}{T} * \lambda_{i}\right) \frac{\frac{\text { bits }}{\mathrm{s}}}{\mathrm{Hz}} .
$$

Fig. 10 shows the measured capacity for all tested systems versus SNR for different K-factors. Fig. 11 depicts the same measured capacity versus K-factor for different SNR values. Fig. 10 clearly shows that system capacity decreases with increasing $\mathrm{K}$-factor, as expected. The decrement is more noticeable for very high SNR values, as it was also expected. As an example, for system B and a SNR $=30 \mathrm{~dB}$, the capacity loss with regard to the case with $K=0.001,1$ and 10000 is 1.6 $\mathrm{bit} / \mathrm{s} / \mathrm{Hz}(7 \%), 6.1 \mathrm{bit} / \mathrm{s} / \mathrm{Hz}$ (26.9\%) and $11 \mathrm{bit} / \mathrm{s} / \mathrm{Hz}$ (48.7\%), respectively. For very high K-factors all systems exhibit the same capacity, as it was also expected. Similarly, the minimum SNR required for achieving a specific system capacity can be extracted from Fig. 11. For example, in order to reach a system B capacity of $10 \mathrm{bits} / \mathrm{s} / \mathrm{Hz}$, a SNR $=15,16.3,20,23.2$ and 25 would be required for K-factors of $0.001,0.1,1,10,100$ and 1000, respectively. Some examples of SNR values required to reach specific capacities at different $\mathrm{K}$-factors are listed in Table II.

For example, if a capacity greater than $15 \mathrm{bit} / \mathrm{s} / \mathrm{Hz}$ is required, this can be done with a SNR $=25 \mathrm{~dB}$ and $K<3$ or a $\mathrm{SNR}=35 \mathrm{~dB}$ and $K<350$. This requirement would not be fulfilled for SNR $<15 \mathrm{~dB}$. Finally, it is also clear from Fig. 11 that for high K-factors and given a specific SNR value, all systems achieve the same capacity. The capacity increase when changing a MIMO system to a different one out of Table I is illustrated in Table III with respect to the K-factor. From this Table it is also extracted that the change from system A to B produce a considerable capacity increase (over 15\%) for $K$-factor $\leq 50$ with SNR $=35 \mathrm{~dB}$. The same change but with 


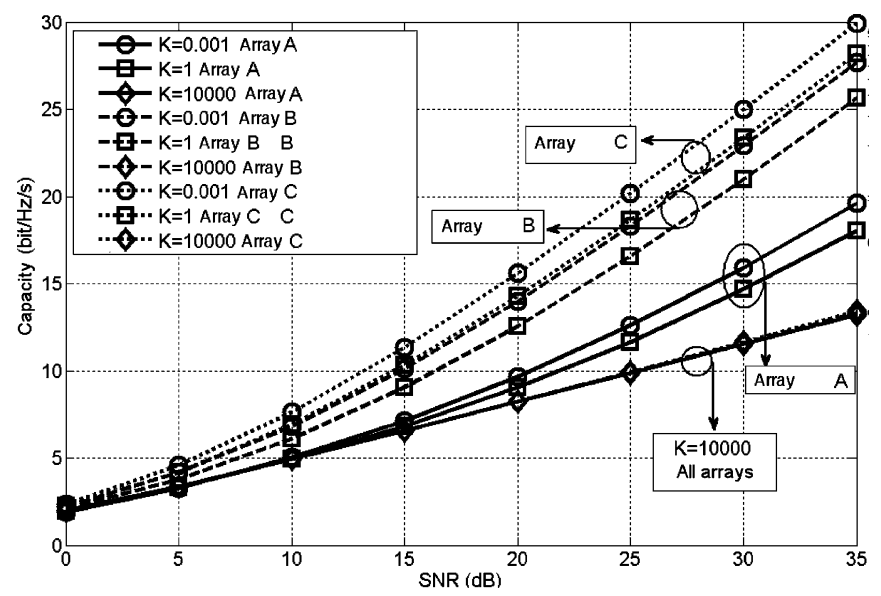

Fig. 10. Measured capacity of all tested systems versus SNR for different K-factors.

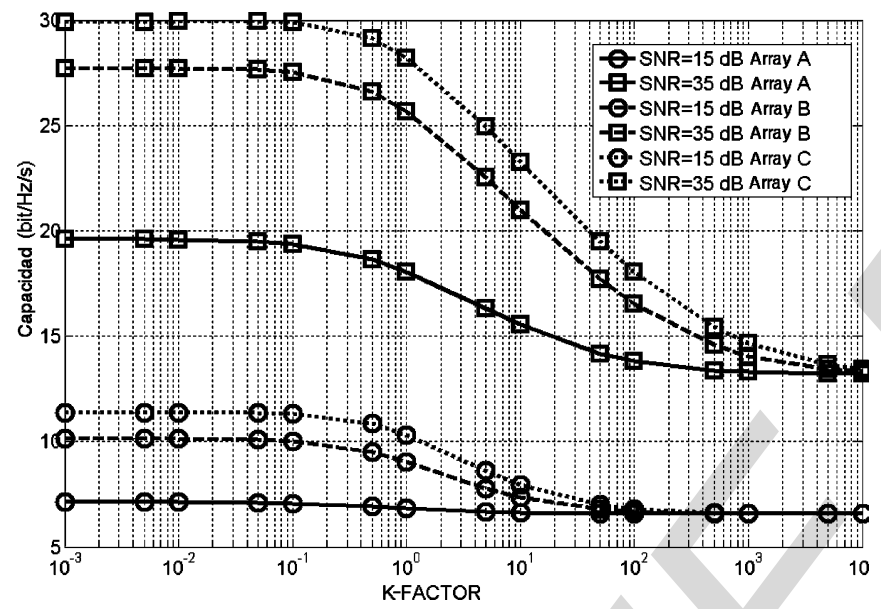

Fig. 11. Measured capacity of all tested systems versus K-factor for different SNR values.

TABLE II

REQUIRED SNR FOR DIFFERENT CAPACITIES

\begin{tabular}{|c|c|c|c|c|c|c|c|c|c|}
\hline K-factor & \multicolumn{3}{|c|}{ K=0.001 } & \multicolumn{3}{c|}{ K=1 } & \multicolumn{3}{c|}{ K=10 } \\
\hline System & A & B & C & A & B & C & A & B & C \\
\hline $\begin{array}{c}\text { Cap. } \\
\text { bits/s/H } \\
\text { z }\end{array}$ & $\begin{array}{c}\text { SNR } \\
(d B)\end{array}$ & $\begin{array}{c}\text { SNR } \\
(d B)\end{array}$ & $\begin{array}{c}\text { SNR } \\
(d B)\end{array}$ & $\begin{array}{c}\text { SNR } \\
(d B)\end{array}$ & $\begin{array}{l}\text { SNR } \\
(d B)\end{array}$ & $\begin{array}{c}\text { SNR } \\
(d B)\end{array}$ & $\begin{array}{c}\text { SNR } \\
(d B)\end{array}$ & $\begin{array}{c}\text { SNR } \\
(d B)\end{array}$ & $\begin{array}{c}\text { SNR } \\
(d B)\end{array}$ \\
\hline 10 & 20.3 & 15 & 13.4 & 22.2 & 16.1 & 14.7 & 25 & 25 & 25 \\
\hline 15 & 28.1 & 21.3 & 19.2 & 30.4 & 23.1 & 20.9 & --- & --- & --- \\
\hline 20 & ---- & 27.4 & 24.9 & ---- & 28.6 & 26.9 & --- & --- & -- \\
\hline
\end{tabular}

$\mathrm{SNR}=15 \mathrm{~dB}$, however, provides for similar capacity increments but this time for $K$-factor $<0.5$. This means that larger spatial separations in MIMO arrays provide a better benefit for high SNR scenarios.

\section{CONCLUSIONS}

Accurate emulation of any Rician-fading environment has been performed with a mode-stirred reverberation chamber for the first time. The new technique allows for greater versatility and a more detailed study of the influence of the K-factor on
TABLE III

CAPACITY INCREASE (\%) WHEN CHANGING MIMO SYSTEMS

\begin{tabular}{|c|c|c|c|c|}
\hline SNR & \multicolumn{2}{|c|}{$15 \mathrm{~dB}$} & \multicolumn{2}{c|}{$35 \mathrm{~dB}$} \\
\hline K-factor & A toB & B to C & A to B & B to C \\
\hline 0.5 & 35.7 & 13.6 & 43.0 & 10.1 \\
\hline 5 & 14.5 & 7.5 & 38.8 & 11.0 \\
\hline 50 & 2.8 & 2.8 & 25.0 & 11.4 \\
\hline 500 & 0 & 0 & 6.5 & 5.4 \\
\hline 5000 & 0 & 0 & 0.7 & 0.7 \\
\hline
\end{tabular}

MIMO performance. Correlation, diversity gain and MIMO capacity have been analyzed for three different MIMO systems under a variety of Rician-fading environments with different K-factors. Different effects have been evaluated. Results confirm previously-published influence of K-factors for outdoor measurements, yet this time with the use of a mode-stirred chamber for the first time. The proposed technique allows for a better study of K-factor influence on MIMO performance for wireless communications systems, avoiding cumbersome outdoor measurements. The results presented in this paper have been employed to extend the measuring capabilities of EMITE Ing second generation mode-stirred reverberation chambers, in addition to the emulation of non-isotropic environments. The technique represents a step forward to the goal of obtaining real-world performance from a mode-stirred reverberation chamber test. The technique is patent protected by EMITE Ing. Future research works include the hybridization of the new technique with some sample selection algorithm so as to further increase accuracy while reducing representative test samples to those important for each specific fading scenario.

\section{REFERENCES}

[1] C. Bruns and R. Vahldieck, "A closer look at reverberation chambers-3D simulation and experimental verification," IEEE Trans. Electromagn. Compat., vol. 47, pp. 612-626, Aug. 2005.

[2] K. Rosengren and P. S. Kildal, "Theoretical study of distributions of modes and plane waves in reverberation chamber for characterization of antennas in multipath environment," Microw. Opt. Technol. Lett., vol. 30, pp. 386-391, 2001.

[3] P. S. Kildal, K. Rosengren, J. Byun, and J. Lee, "Definition of effective diversity gain and how to measure it in a reverberation chamber," Microw. Opt. Technol. Lett., vol. 34, no. 1, pp. 56-59, Jul. 2002.

[4] P. S. Kildal and K. Rosengren, "Correlation and capacity of MIMO systems and mutual coupling, radiation efficiency, and diversity gain of their antennas: Simulations and measurement in a reverberation chamber," IEEE Commun. Mag., pp. 104-112, Dec. 2004.

[5] L. Greenstein, S. Ghassemzadeh, V. Erceg, and D. G. Michelson, "Theory, experiments, and statistical models," presented at the WPMC'99 Conf., Amsterdam, Sep. 1999.

[6] E. Green, "Radio link design for microcellular systems," BT Tech. J., vol. 8, no. 1, pp. 85-96, 1990.

[7] V. R. Anreddy and M. A. Ingram, "Capacity of measured Ricean and Rayleigh indoor MIMO channels at $2.4 \mathrm{GHz}$ with polarization and spatial diversity," in Proc. IEEE Wireless Communications and Networking Conf. (WCNC'06), Apr. 2006, vol. 2, pp. 946-951.

[8] J. F. Valenzuela-Valdés, A. M. Martínez-González, and D. A. SánchezHernández, "Emulation of MIMO non-isotropic fading environments with reverberation chambers," IEEE Antennas Wireless Propag. Lett., vol. 7, pp. 325-328, 2008

[9] Z. Yun and M. F. Iskander, "MIMO capacity for realistic wireless communications environments," in Proc. IEEE Antennas and Propagation Society Int. Symp., Jun. 2004, pp. 1231-1234.

[10] P. Hallbjorner and K. Madsen, "Terminal antenna diversity characterization using mode stirred chamber," Electron. Lett., vol. 37, no. 5, pp. 273-274, Mar. 2001. 
[11] J. F. Valenzuela-Valdés et al., "Evaluation of true polarization diversity for MIMO systems," IEEE Trans. Antennas Propag., vol. 57, pp. 2746-2755, Sep. 2009.

[12] J. D. Sánchez-Heredia, M. Grudén, J. F. Valenzuela-Valdés, and D. A. Sánchez-Hernández, "Sample selection method for arbitrary fading emulation using mode-stirred chambers," IEEE Antennas and Wireless Propag. Lett., 2010, to be published.

[13] C. L. Holloway, D. A. Hill, J. M. Ladbury, P. F. Wilson, G. Koepke, and J. Coder, "On the use of reverberation chambers to simulate a Rician radio environment for the testing of wireless devices," IEEE Trans. Antennas Propag., vol. 54, pp. 3167-3177, Nov. 2006.

[14] M. Kang and M. S. Alouini, "Capacity of MIMO Rician channels," IEEE Trans. Wireless Commun., vol. 5, pp. 112-122, Jan. 2006.

[15] C. Chen-Nee, J. M. Kahn, and D. Tse, "Capacity of multi-antenna array systems in indoor wireless environment," in Proc. IEEE Global Telecommunications Conf. GLOBECOM 98, 1998, vol. 4, pp. 1894-1899.

[16] D. Chizhik, G. J. Foschini, and R. A. Valenzuela, "Capacities of multi-element transmit and receive antennas: Correlations and keyholes," Electron. Lett., vol. 36, no. 13, pp. 1099-1100, Jun. 2000.

[17] K. I. Pedersen, J. B. Andersen, J. P. Kermoal, and P. Mogensen, "A stochastic multiple-input-multiple-output radio channel model for evaluation of space-time coding algorithms," in Pro. IEEE Vehicular Technology Conf., Sep. 2000, vol. 2, pp. 893-897.

[18] J. P. Kermoal, L. Schumacher, K. I. Pedersen, P. E. Mogensen, and F. Frederiksen, "A stochastic MIMO radio channel model with experimental validation," IEEE J. Sel. Areas Commun., vol. 20, pp. 1211-1226, Aug. 2002.

[19] D. Chizhik, J. Ling, P. W. Wolniansky, R. A. Valenzuela, N. Costa, and K. Huber, "Multiple-input-Multiple-output measurements and modelling in Manhattan," IEEE J. Sel. Areas Commun., vol. 21, pp. 321-331, 2003.

[20] W. Weichselberger, M. Herdin, H. Ozcelik, and E. Bonek, "A stochastic MIMO channel model with joint correlation of both link ends," IEEE Trans. Wireless Commun., vol. 5, pp. 90-100, Jan. 2006.

[21] G. Foschini and M. Gans, "On limits of wireless communication in a fading environment when using multiple antennas," Wireless Personal Commun., pp. 311-335, Mar. 1998.

[22] E. Telatar, "Capacity of multi-antenna Gaussian channels," Eur. Trans. Telecommun., vol. 10, no. 6, pp. 585-595, Nov./Dec. 1999.

[23] J. Winters, "On the capacity of radio communication systems with diversity in a Rayleigh fading environment," IEEE J. Sel. Areas Commun., vol. 5, pp. 871-878, Jun. 1987.

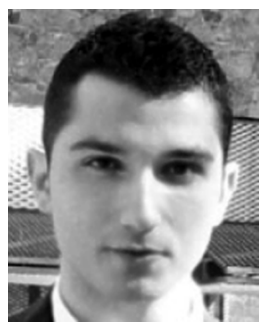

Juan D. Sánchez-Heredia was born in Lorca, Spain. He received the Telecommunication Engineering Degree from the Universidad Politécnica de Cartagena, Spain, in 2009, and is working toward the Masters degree in information technologies at the Universidad de Murcia, Spain.

In 2007, he worked at General Electric (Cartagena), and was involved in several projects in relation with the network infrastructure. In 2009, he joined the Department of Information Technologies and Communications, Universidad Politécnica de Cartagena, Spain. His current research areas cover MIMO communications, multimode-stirred chambers and electromagnetic dosimetry.

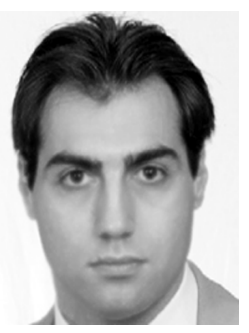

Juan F. Valenzuela-Valdés was born in Marbella, Spain. He received the Degree in Telecommunications Engineering from the Universidad de Malaga, Spain, in 2003 and the Ph.D. from Universidad Politécnica de Cartagena, in May 2008.

In 2004, he worked at CETECOM (Malaga). In 2004, he joined the Department of Information Technologies and Communications, Universidad Politécnica de Cartagena, Spain. In 2007, he joined EMITE Ing as Head of Research. His current research areas cover MIMO communications, multimode-stirred chambers and SAR measurements.

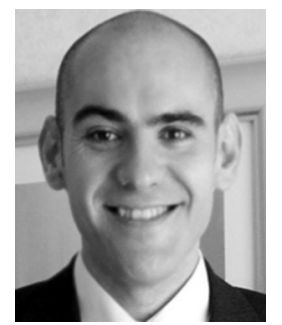

Antonio M. Martínez-González received the Dipl.-Ing. degree in telecommunications engineering from the Universidad Politécnica de Valencia, Spain, in 1998 and the Ph.D. degree from the Universidad Politécnica de Cartagena, Spain, in 2004

From 1998 until September 1999, he was employed as a Technical Engineer at the Electromagnetic Compatibility Laboratory, Universidad Politécnica de Valencia, where he developed assessment activities and compliance certifications with European directives related with immunity and emissions to electromagnetic radiation from diverse electrical, electronic and telecommunication equipment. Since September 1999, he has been an Associate Professor at the Universidad Politécnica de Cartagena. At present, his research interest is focused on electromagnetic dosimetry, radioelectric emissions and mode stirred chambers applied to MIMO systems. In December 2006, he was one of the founders of EMITE Ing, a technological spin-off company founded by telecommunication engineers and doctors of the Microwave, Radiocommunications and Electromagnetism Research Group (GIMRE), Technical University of Cartagena (Spain).

Prof. Martínez-González was awarded with the Spanish National Prize from Foundation Airtel and Colegio Oficial de Ingenieros de Telecomunicación de España for the best final project on mobile communications in 1999. In 2006 and 2008, the second i-patentes prize for innovation and technology transfer in the Region of Murcia (Spain) was awarded to the founders of EMITE Ing.

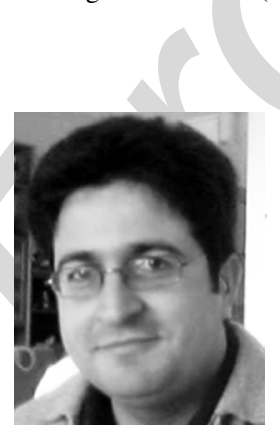

David A. Sánchez-Hernández (M'00-SM'06) received the Dipl.-Ing. degree in telecommunications engineering from the Universidad Politécnica de Valencia, Spain, in 1992 and the Ph.D. degree from King's College, University of London, London, U.K., in early 1996.

From 1992 to 1994 , he was employed as a Research Associate for The British Council-CAM, King's College London, where he worked on active and dual-band microstrip patch antennas. In 1994, he was appointed EU Research Fellow at King's College London, working on several joint projects at 18,38 and $60 \mathrm{GHz}$ related to printed and integrated antennas on $\mathrm{GaAs}$, microstrip antenna arrays, sectorization and diversity. In 1997, he returned to Universidad Politécnica de Valencia, Spain, where was co-leader of the Antennas, Microwaves and Radar Research Group and the Microwave Heating Group. In early 1999, he received the Readership from Universidad Politécnica de Cartagena, and was appointed Vice Dean of the School for Telecommunications Engineering and Leader of the Microwave, Radiocommunications and Electromagnetism Engineering Research Group. In late 1999, he was appointed Vice Chancellor for Innovation \& Technology Transfer at the Universidad Politécnica de Cartagena and a member of several Foundations and Societies for promotion of R\&D in the Autonomous Region of Murcia, in Spain. In May 2001, he was appointed official advisor in technology transfer and a member of The Industrial Advisory Council of the Autonomous Government of the Region of Murcia, Spain. In May 2003 he was appointed Head of Department and, in June 2009, he received the Chair at the Universidad Politécnica de Cartagena, Spain. He has published three international books, over 45 scientific papers and over 90 conference contributions, and is a reviewer of several international journals. He holds six patents. His current research interests encompass all aspects of the design and application of printed multi-band antennas for mobile communications, electromagnetic dosimetry issues and MIMO techniques for wireless communications.

Dr. Sánchez-Hernández is a Chartered Engineer (CEng), IET Fellow, IEEE Senior Member, CENELEC TC106X member, and is the recipient of the R\&D J. Langham Thompson Premium, awarded by the Institution of Engineering and Technology, the i-Patentes award to innovation and technology transfer, the Emprendedor XXI award to innovative entrepreneurship, granted by the Spanish National Innovation Entity (ENISA), as well as other national and international awards. 